\title{
CD62L+ NKT cells have prolonged persistence and antitumor activity in vivo
}

\author{
Gengwen Tian, ${ }^{1}$ Amy N. Courtney, ${ }^{1}$ Bipulendu Jena, ${ }^{2}$ Andras Heczey, ${ }^{1}$ Daofeng Liu, ${ }^{1}$ Ekaterina Marinova, ${ }^{1}$ Linjie Guo, ${ }^{1}$ Xin Xu, ${ }^{1}$ \\ Hiroki Torikai, ${ }^{2}$ Qianxing Mo, ${ }^{3}$ Gianpietro Dotti, ${ }^{4}$ Laurence J. Cooper, ${ }^{2}$ and Leonid S. Metelitsa ${ }^{1,4}$ \\ 'Department of Pediatrics and Department of Pathology and Immunology, Baylor College of Medicine, Houston, Texas, USA. ²Division of Pediatrics, MD Anderson Cancer Center, Houston, Texas, USA. \\ ${ }^{3}$ Division of Biostatistics, Dan L. Duncan Cancer Center, Department of Medicine, and ${ }^{4}$ Center for Cell and Gene Therapy, Department of Medicine, Baylor College of Medicine, Houston, Texas, USA.
}

\begin{abstract}
Vo24-invariant natural killer T cells (NKTs) localize to tumors and have inherent antitumor properties, making them attractive chimeric antigen receptor (CAR) carriers for redirected cancer immunotherapy. However, clinical application of CAR-NKTs has been impeded, as mechanisms responsible for NKT expansion and the in vivo persistence of these cells are unknown. Here, we demonstrated that antigen-induced expansion of primary NKTs in vitro associates with the accumulation of a CD62L subset and exhaustion of CD62L- cells. Only CD62 $\mathrm{L}^{+} \mathrm{NKTs}$ survived and proliferated in response to secondary stimulation. When transferred to immune-deficient NSG mice, CD62L+ NKTs persisted 5 times longer than CD62L- NKTs. Moreover, CD62L' cells transduced with a CD19-specific CAR achieved sustained tumor regression in a B cell lymphoma model. Proliferating CD62L+ cells downregulated or maintained CD62L expression when activated via $\mathrm{T}$ cell receptor alone or in combination with costimulatory receptors. We generated HLA ${ }^{\text {null }} \mathrm{K} 562$ cell clones that were engineered to express CD1d and costimulatory ligands. Clone B-8-2 (HLA ${ }^{\text {null }} C D 1 d^{\text {med }} C D 86^{\text {high }}$ 4-1BBL ${ }^{\text {med }} O X 4 \mathrm{OL}^{\text {high }}$ ) induced the highest rates of NKT expansion and CD62L expression. B-8-2-expanded CAR-NKTs exhibited prolonged in vivo persistence and superior therapeutic activities in models of lymphoma and neuroblastoma. Therefore, we have identified CD62L as a marker of a distinct NKT subset endowed with high proliferative potential and have developed artificial antigen-presenting cells that generate CD62L-enriched NKTs for effective cancer immunotherapy.
\end{abstract}

\section{Introduction}

Type I NKT cells (NKTs) are an evolutionarily conserved subset of innate lymphocytes that express invariant T cell receptor (TCR) $\alpha$-chain V $\alpha 24-\mathrm{J} \alpha 18$ and react to self- or microbial-derived glycolipids presented by the monomorphic HLA class I-like molecule CD1d (1-4). The potential importance of NKTs for tumor immunity and immunotherapy has been demonstrated in multiple models of cancer in mice and in early-stage clinical trials in cancer patients (5-10). In contrast to T cells, NKTs effectively traffic to the tumor site and can mediate antitumor responses via either direct killing of CD1 $\mathrm{d}^{+}$tumor cells, inhibition of tumor-supportive macrophages, or transactivation of NK cells (11). Several studies

Note regarding evaluation of this manuscript: Manuscripts authored by scientists associated with Duke University, The University of North Carolina at Chapel Hill, Duke-NUS, and the Sanford-Burnham Medical Research Institute are handled not by members of the editorial board but rather by the science editors, who consult with selected external editors and reviewers.

Conflict of interest: G. Tian, A.N. Courtney, A. Heczey, D. Liu, G. Dotti, and L.S. Metelitsa are co-inventors on pending patent applications that relate to the use of NKT cells in cancer immunotherapy. Some of the technology used in the generation of the artificial antigen-presenting cells for NKT cells was advanced through research conducted at the MD Anderson Cancer Center under the direction of L.J. Cooper. In January 2015, the technology was licensed for commercial application to Ziopharm Oncology Inc. and Intrexon Corp. in exchange for equity interests in each of these companies for which L.J. Cooper and B. Jena are entitled to receive a portion. On May 7, 2015, L.J. Cooper was appointed as the Chief Executive Officer at Ziopharm. L.J. Cooper is now a Visiting Scientist at MD Anderson. B. Jena is a co-inventor of anti-Id CD19-CAR mAb (patent pending) Submitted: June 29, 2015; Accepted: March 22, 2016.

Reference information: / Clin Invest. 2016;126(6):2341-2355. doi:10.1172/JCI83476. have revealed strong positive associations between the numbers of tumor-infiltrating or circulating NKTs and improved disease outcome in patients with diverse tumor types $(6,12-15)$. Conversely, tumor progression is often accompanied by a decrease in NKT number or functional activity (16), or the downregulation of CD1d expression on malignant cells (17). To counteract these tumor escape mechanisms, we developed methods to expand primary human NKTs to clinical scale ex vivo and to redirect their cytotoxicity against tumor cells via transgenic expression of chimeric antigen receptors (CARs) (18). Similar to the observations reported in CAR-T cell clinical trials $(19,20)$, there is a strong correlation between the antitumor efficacy and in vivo persistence of CAR-NKT products in a xenogenic tumor model (18). However, the mechanisms that govern ex vivo expansion and subsequent in vivo persistence of human NKTs remain largely unknown, impeding rational design of NKT-based cancer immunotherapy.

Recent global transcriptional profiling studies demonstrated that NKTs, though they share properties with T and NK cells, are a distinct population of lymphocytes (21). In the mouse, the developmental program and functional differentiation of NKTs have been characterized quite extensively during the last decade, as summarized in recent reviews $(4,22)$. Several key features of murine NKTs have also been confirmed in their human counterparts. Both in mice and in humans, NKTs diverge from $\mathrm{T}$ cells at the stage of $\mathrm{CD}^{+}{ }^{+} \mathrm{CD} 8^{+}$(double-positive, DP) thymocytes. Unlike $\mathrm{T}$ cells, which are positively selected by thymic epithelial cells, NKTs are selected by CD1d-expressing DP thymocytes (23). The expression of promyelocytic leukemia zinc finger transcription 
factor (PLZF) immediately after positive selection enables intrathymic expansion and effector-memory-like differentiation of NKTs (24). Peripheral NKTs are long-lived lymphocytes, and their post-thymic maintenance largely depends on slow IL-15-mediated homeostatic proliferation $(25,26)$. In human peripheral blood, NKTs are divided into 2 major functional subsets based on CD4 expression: $\mathrm{CD}^{+}$and CD4- (mostly CD8/CD4-double-negative, $\mathrm{DN})$ (27). The CD $4^{+}$subset is highly enriched in neonate NKTs and undergoes fewer homeostatic divisions compared with the CD4 subset in adults (26), suggesting that CD $4^{+}$NKTs could contribute to the long-term persistence of adoptively transferred therapeutic NKTs under certain conditions. However, ex vivo expansion of human NKTs in response to antigenic stimulation, e.g., with $\alpha$-galactosylceramide ( $\alpha$ GalCer), produces similar numbers of $\mathrm{CD}^{+}$and DN NKTs (28). NKTs also exhibit an NK-like linear differentiation with acquisition of CD161 and then CD56 expression. Like in T cells, the expression of CD56 is associated with terminal differentiation and the loss of proliferative potential (29).

In contrast to peripheral $\mathrm{T}$ cells, which have a well-established developmental hierarchy from naive to central-memory to effector-memory to terminal effector cells (30), NKTs are broadly described as cells with "activated memory" phenotype without the naive state $(31,32)$. In cord blood, the majority of NKTs are CD $4^{+}$ and coexpress CD45RO with CD62L and CCR7 without immediate effector function $(26,31,33)$, thus resembling central-memory CD4 $\mathrm{T}$ cells. In adult peripheral blood, NKTs are equally split between $\mathrm{CD}^{+}$and $\mathrm{CD}^{-}$subsets (albeit with significant interindividual variability). Adult NKTs lack a clear demarcation between "memory" and "effector" states, as they variably express memory markers and have immediate effector functions such as cytokine production and cytotoxicity $(26,33)$. The majority of adult NKTs even in the elderly express CD28 (34), making them distinct from terminally differentiated $\mathrm{T}$ effector cells (35).

Recent reports have demonstrated that $\mathrm{CD} 62 \mathrm{~L}^{+}$central memory $\mathrm{T}$ cells have stem cell properties and superior therapeutic activity in cell therapy products (36-38). The functional significance of CD62L expression in NKTs remains unknown. In this study, we found that the $\mathrm{CD} 62 \mathrm{~L}^{+}$subset is required for NKT ex vivo expansion and in vivo persistence. Importantly, when engineered to express CD19-specific CAR (CAR.CD19), CD62L but not CD62L CAR.CD19 NKTs produced sustained tumor regression in a B cell lymphoma model in NOD/SCID/IL-2R $\gamma$ (null) (NSG) mice. We also found that $\mathrm{CD} 62 \mathrm{~L}^{+} \mathrm{NKT}$ s could be maintained during ex vivo expansion when provided with certain costimulatory ligands. With this knowledge, we engineered costimulatory artificial antigen-presenting cells (aAPCs) that can be used to generate NKTs and CARNKTs with superior therapeutic activity in patients with cancer.

\section{Results}

CD62L $L^{+}$cells accumulate in culture upon antigenic stimulation of primary NKTs. Previous studies that compared the phenotype of human NKTs in adult peripheral blood with that in cord blood observed much higher proportions of $\mathrm{CD}^{+}$and $\mathrm{CD}^{2} 2 \mathrm{~L}^{+} \mathrm{NKTs}$ in neonates (refs. 26, 31, 33; and Supplemental Figure 1A; supplemental material available online with this article; doi:10.1172/JCI83476DS1). The prevalence of $\mathrm{CD} 4{ }^{+} \mathrm{CD} 62 \mathrm{~L}^{+} \mathrm{NKTs}$ in cord blood suggests that the expression of CD4 or/and CD62L marks a subset of NKTs that has superior developmental potential and could support ex vivo expansion of NKTs for therapeutic applications. To test this hypothesis, we performed immunophenotyping of primary NKTs immediately after isolation from peripheral blood and at different time intervals in culture after stimulation with $\alpha \mathrm{GalCer}$, which consistently produced higher frequency and absolute numbers of NKTs compared with the use of a T cell expansion protocol based on CD3/ CD28 stimulation (Supplemental Figure 2). Despite a notable interindividual variability of CD62L expression in freshly isolated NKTs, we observed a striking accumulation of $\mathrm{CD} 62 \mathrm{~L}^{+}$fraction of NKTs from $33.63 \% \pm 27.62 \%$ in freshly isolated NKTs to $69.92 \%$ $\pm 10.57 \%$ on day 12 of the culture $(P<0.001$, Figure $1, A$ and $B)$. Although CD62L was more frequently expressed on CD4 ${ }^{+}$NKTs both before and after culture, the accumulation of $\mathrm{CD}^{2} 2 \mathrm{~L}^{+}$cells could not be explained by a preferential expansion of $\mathrm{CD}^{+} \mathrm{NKTs}$. Indeed, at the end of a 12-day culture, the frequency of $\mathrm{CD} 62 \mathrm{~L}^{+}$, $\mathrm{CD}^{+}$, and $\mathrm{CD} 62 \mathrm{~L}^{+} \mathrm{CD} 4^{+}$NKTs increased $3.9 \pm 1.8$-fold, $1.9 \pm 0.7-$ fold, and 3.8 \pm 1.9 -fold, respectively. Consistent with these results, we also observed an enrichment of the $\mathrm{CD} 2 \mathrm{~L}^{+} \mathrm{CD} 4^{-}$subset at day 12 compared with day 0 (Supplemental Figure 1B). Further multiparameter characterization of NKTs showed that CD62L was often coexpressed with CCR7 before culture, but CCR7 expression was progressively lost during culture (Supplemental Figure 1C). We also found that nearly all NKTs expressed both CD27 and CD28 before culture. While CD27 was downregulated in about half of NKTs by day 12 of culture irrespective of CD62L status, CD28 expression remained intact (Supplemental Figure 1C).

CD62 $\mathrm{L}^{-}$cells expressed higher levels of CD161 and CD56 (NK-like differentiation), but a lower level of IL-7R $\alpha$ (Supplemental Figure 1D). While freshly isolated NKTs rarely expressed exhaustion markers (PD-1, LAG-3, or TIM-3) in either the CD62L $\mathrm{L}^{+}$ or the $\mathrm{CD}^{2} 2 \mathrm{~L}^{-}$subset, the $\mathrm{CD} 62 \mathrm{~L}^{-}$subset preferentially expressed PD-1 and TIM-3 at day 12 of NKT culture $(P=0.0043,0.0184$, Figure $1 C$ ). Moreover, immune-related gene expression analysis (nCounter platform) of CD62 $\mathrm{L}^{+}$and CD62L- NKTs sorted on day 12 revealed mRNA upregulation of genes associated with $\mathrm{T}$ cell survival/memory (e.g., LEF1, S1PR1, IL7RA, IL21R) in CD62L $\mathrm{L}^{+}$ NKTs and with exhaustion/terminal differentiation in $\mathrm{CD}^{2} \mathrm{~L}^{-}$ NKTs (e.g., PD1, LAG3, TIM3, CD244, CD161, CD56; Figure 1D and Supplemental Table 1). The transcription factor lymphoid enhancer factor 1 (LEF1) was the top immune-related gene overexpressed in $\mathrm{CD} 6 \mathrm{~L}^{+}$compared with CD62L- NKTs. Intracellular flow cytometry analysis demonstrated that CD62L $\mathrm{L}^{+}$NKTs uniformly expressed LEF1, whereas a major fraction of CD62 $\mathrm{L}^{-}$cells was LEF1-negative. Since LEF1 was recently shown to mediate expansion of murine NKT precursors in part via transcriptional activation of GATA3 gene expression (39), we analyzed the level of GATA3 protein in human NKTs in relation to LEF1 and CD62L levels. We found that GATA3 expression strongly correlated with the expression of LEF1 and CD62L in human NKTs (Supplemental Figure 1E). Interestingly, CD62 $\mathrm{L}^{+}$and CD62 $\mathrm{L}^{-}$NKTs expressed the same level of PLZF, a transcriptional master regulator of NKT functional differentiation (21). Thus, the CD62 $\mathrm{L}^{+}$subset predominantly accumulates in culture upon antigenic stimulation of primary NKTs, and loss of CD62L expression is associated with NKlike terminal differentiation, exhaustion, and downregulation of pro-proliferative transcriptional regulators. 
A

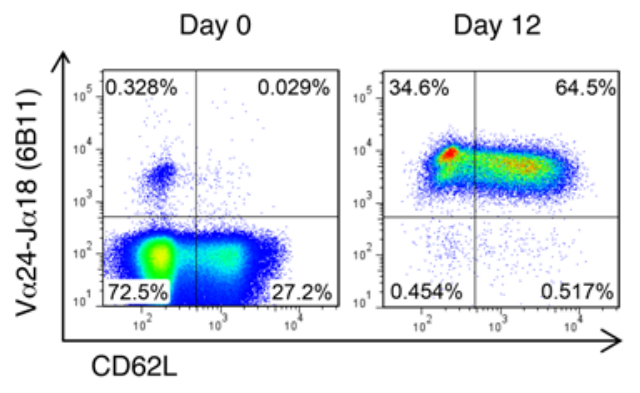

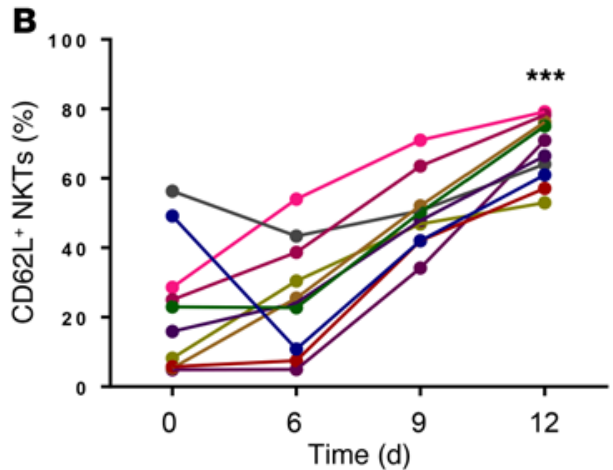

Time (d)

C

Day 0
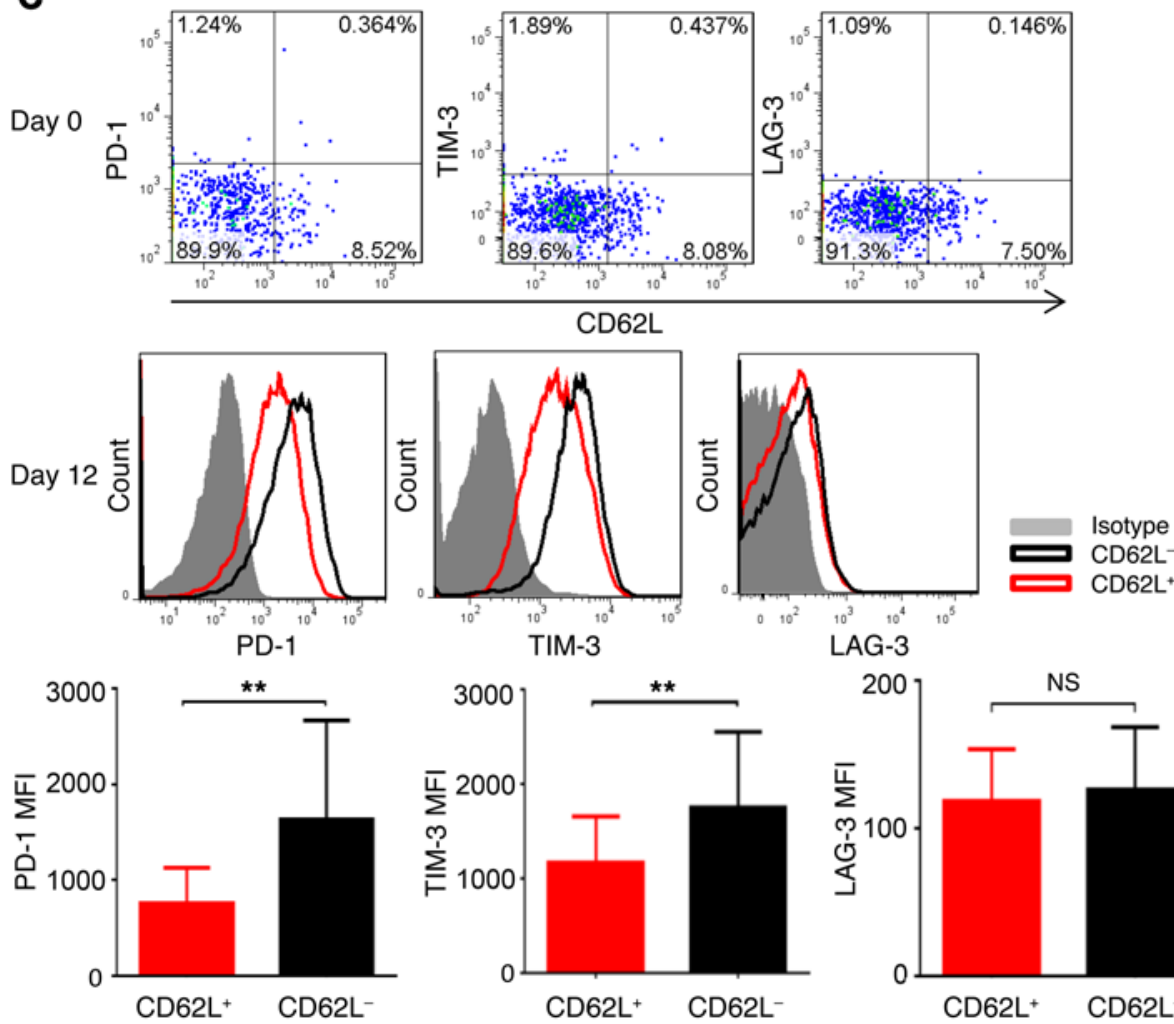

LAG-3
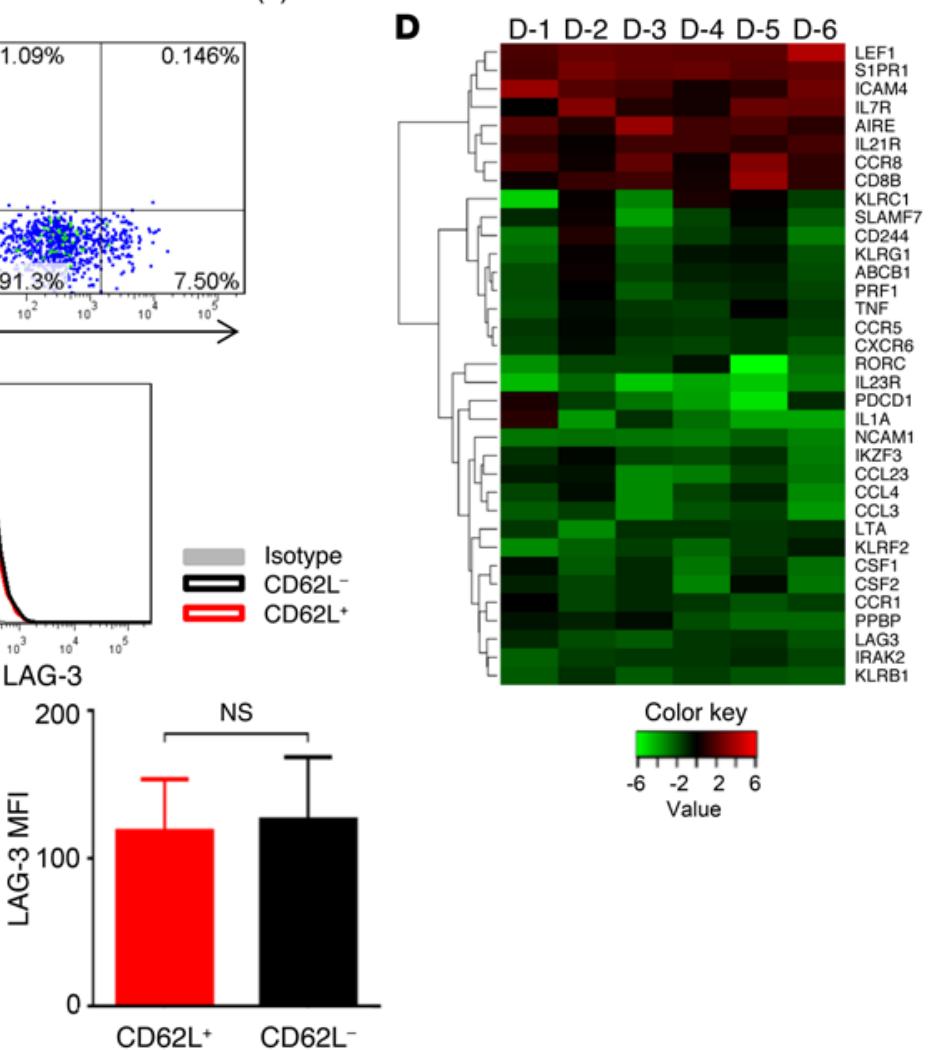

Figure 1. Accumulation of CD62L+ subset in culture after in vitro antigenic stimulation of primary NKTs. (A) CD62L expression was examined by FACS in primary NKTs from freshly isolated PBMCs (day 0 ) and 12 days after stimulation with $\alpha$ GalCer and in vitro expansion in culture $(n=10)$. (B) Kinetics of CD62L expression in NKTs at the indicated intervals after primary stimulation (as in A) from individual donors ( $n=10$ ). (C) Expression of PD-1, TIM-3, and LAG-3 on NKT surface was measured by FACS on days 0 and 12 (top panel). Representative plots from 1 of 4 donors (middle panel) or mean \pm SD of mean fluorescence intensity (MFI) for all donors ( $n=4$, bottom panel). (D) On day 12 after primary stimulation, NKTs were magnetically sorted into CD62 $\mathrm{L}^{+}$ and CD62L- subsets followed by RNA isolation and gene expression analysis using Human Immunology Panel version 2 and $\mathrm{nCounter} \mathrm{Analysis} \mathrm{System}$ (NanoString). The heat map shows the $\log _{2}$ fold changes (CD62 $/ \mathrm{CD}^{2} \mathrm{~L}^{-}$) of genes with $P$ values less than 0.02 and average fold change greater than 2 . Data were generated from 6 NKT donors (12 paired samples). ${ }^{* *} P<0.01,{ }^{* *} P<0.001$, paired $t$ test.

CD62L $L^{+}$and CD62L- NKTs have comparable cytotoxicity, but the CD62L subset is the main source of cytokines. We magnetically sorted NKTs from primary culture into $\mathrm{CD}_{62} \mathrm{~L}^{+}$and $\mathrm{CD} 62 \mathrm{~L}^{-}$subsets and examined their functional properties. Figure 2A demonstrates that either both subsets were equally cytotoxic (3 of 6 donors) or $\mathrm{CD}^{2} 2 \mathrm{~L}^{-} \mathrm{NKTs}$ were more cytotoxic than $\mathrm{CD}^{-} \mathrm{L}^{+} \mathrm{NKTs}$ (3 of 6 donors) against $\mathrm{CD} 1 \mathrm{~d}^{+} \mathrm{DAOY}$ medulloblastoma cells when the target cells were pulsed with $\alpha$ GalCer. The analysis of cytokine production in $\alpha$ GalCer-stimulated NKTs revealed much higher levels of both IFN- $\gamma$ and IL-4 production by CD62L $\mathrm{L}^{+}$compared with CD62L- subsets $(P<0.001$, Figure $2 \mathrm{~B}) . \mathrm{CD}^{-} \mathrm{L}^{+}$cells exhibited a
Th0-like polarization (a balanced production of IFN- $\gamma$ and IL-4, typical of the whole population of peripheral blood NKTs), whereas the polarization profile of $\mathrm{CD}^{2} 2 \mathrm{~L}^{-}$cells could not be determined unambiguously because of the low absolute amounts of each cytokine. Despite strong upregulation of IL23R mRNA expression in the CD62L' subset as determined by the nCounter analysis (Figure 1D; a potential Th17 polarization), we detected neither IL-23R protein expression on the cell surface of NKTs by FACS nor production of IL-17 upon TCR-stimulation by ELISA (data not shown).

$C D 62 L^{+}$NKTs have superior survival and proliferative capacity upon secondary stimulation. To examine whether the accumula- 

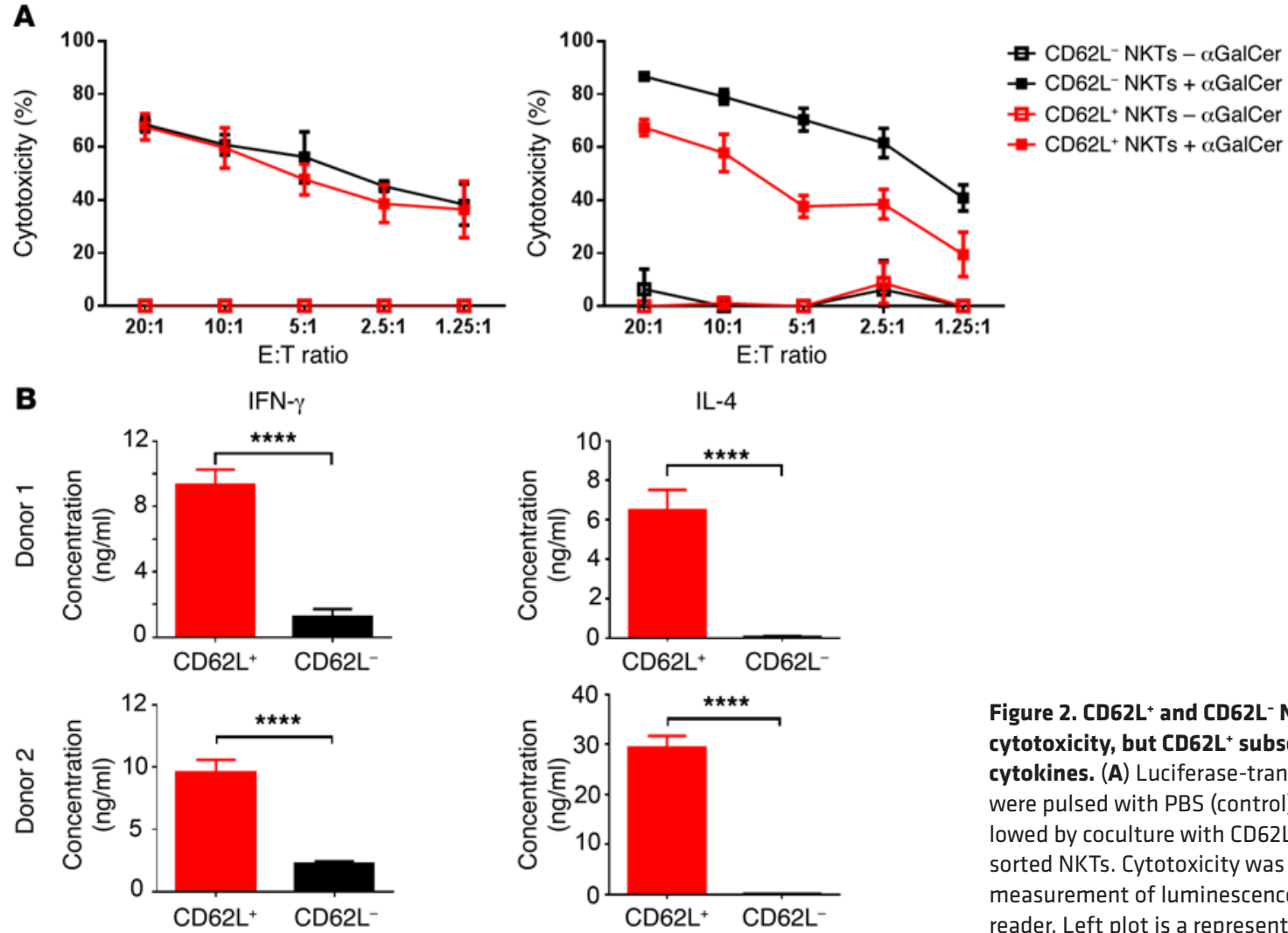

Figure 2. CD62L+ and CD62L- NKTs have comparable cytotoxicity, but CD62L+ subset is the main source of cytokines. (A) Luciferase-transduced CD1d ${ }^{+}$DAOY cells were pulsed with PBS (control) or $\alpha$ CalCer overnight followed by coculture with $\mathrm{CD}^{2} 2 \mathrm{~L}^{+}$or $\mathrm{CD} 62 \mathrm{~L}^{-}$magnetically sorted NKTs. Cytotoxicity was analyzed after 4 hours by measurement of luminescence intensity with a plate reader. Left plot is a representative of 3 donors (in triplicates) with no difference in cytotoxicity between
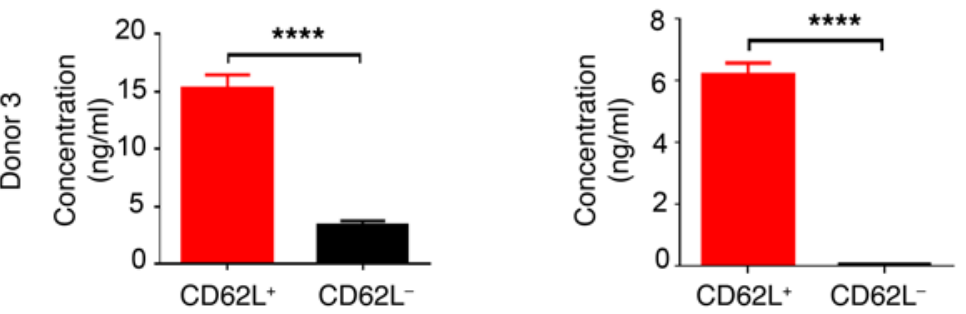
NKT subsets. Shown is mean $\pm S D, n=3$. Right plot is a representative of 3 donors (in triplicates) with a significant difference in cytotoxicity between NKT subsets. Shown is mean $\pm S D, n=3$. (B) The concentrations of IFN- $\gamma$ and IL-4 were measured in 24-hour supernatants of $\alpha$ GalCer-stimulated CD62L+ or CD62L- NKTs by the Luminex assay in 3 independent experiments with NKTs from 3 donors. ${ }^{* * *} P<0.0001$, paired $t$ test.

tion of $\mathrm{CD}^{2} 2 \mathrm{~L}^{+}$NKTs during in vitro expansion is due to their preferential survival or proliferation in response to antigenic stimulation, we measured their rate of cell death and proliferation after stimulation of the sorted cells with APCs that had been pulsed with $\alpha$ GalCer. On day 3 after stimulation, $31 \% \pm 21 \%$ and $74 \% \pm 7.5 \%$ of $\mathrm{CD}^{2} 2 \mathrm{~L}^{+}$and $\mathrm{CD} 62 \mathrm{~L}^{-} \mathrm{NKTs}$ underwent apoptosis, respectively (Figure $3 \mathrm{~A})$. We also observed a much greater rate of proliferation in $\mathrm{CD} 62 \mathrm{~L}^{+}$versus $\mathrm{CD} 62 \mathrm{~L}^{-}$subsets as measured by CFSE dilution on day 6 (Figure 3B). Moreover, the majority of cells that survived and proliferated in the CD62L- group expressed CD62L, suggesting that these cells were progenies of a small subset of $\mathrm{CD} 62 \mathrm{~L}^{+}$cells in the original CD62 $\mathrm{L}^{-}$fraction. Consistent with these results, we observed a striking difference in the numbers of NKTs generated after a 6-day culture of sorted CD62 $\mathrm{L}^{+}$versus CD62L- NKTs with IL-2 alone or with TCRstimulation. Indeed, Figure 3C (top panel) demonstrates that CD62 $\mathrm{L}^{+}$cells underwent 2.5- and 8-fold numeric expansion with IL-2 alone or with TCR-stimulation, respectively. In contrast, CD62 $\mathrm{L}^{-} \mathrm{NKT}$ failed to expand in either condition. Although the degree of NKT proliferation in response to antigenic stimulation varied from donor to donor, the $\mathrm{CD} 62 \mathrm{~L}^{-}$subset contributed little or nothing to NKT expansion in all 5 tested donors (Figure 3C, bottom panel). Therefore, $\mathrm{CD}_{2} \mathrm{~L}^{+} \mathrm{NKT}$ s survive and proliferate in response to antigenic stimulation and are responsible for NKT numeric expansion in culture.

$C D 62 L^{+}$subset is responsible for in vivo persistence and therapeutic activity of NKTs. To determine the role of the CD62L $\mathrm{L}^{+}$subset in the in vivo persistence of adoptively transferred NKTs, we transduced NKTs with firefly luciferase and magnetically sorted them into $\mathrm{CD} 62 \mathrm{~L}^{+}$and $\mathrm{CD} 62 \mathrm{~L}^{-}$subsets. We then adoptively transferred the sorted cells to NSG mice. Longitudinal bioluminescent imaging demonstrated that the signal from CD62 $\mathrm{L}^{-}$cells could be detected until day 2, whereas $\mathrm{CD}_{62} \mathrm{~L}^{+}$cells remained detectable up to day $10(P<0.001$, Figure $4, A$ and $B)$. Next, we compared the in vivo therapeutic potential of $\mathrm{CD}^{2} 2 \mathrm{~L}^{+}$and $\mathrm{CD} 62 \mathrm{~L}^{-} \mathrm{NKT}$ subsets in a model of CAR-redirected immunotherapy for lymphoma. NKTs were transduced with a CD19-specific CAR containing 4-1BB costimulatory endodomain (CAR.CD19, Supplemental Figure 3) followed by sorting into $\mathrm{CD} 2 \mathrm{~L}^{+}$and $\mathrm{CD} 62 \mathrm{~L}^{-}$subsets. NSG mice were i.v. injected with luciferase-transduced CD19+ Daudi lymphoma cells and 4 days later were divided into 2 groups to receive CD62L $\mathrm{L}^{+}$or CD62L- CAR.CD19 NKTs. Both CD62L $\mathrm{L}^{+}$and CD62L- CAR.CD19 NKTs prolonged the survival of treated animals compared with untreated control $(P<0.001)$. Importantly, 
A
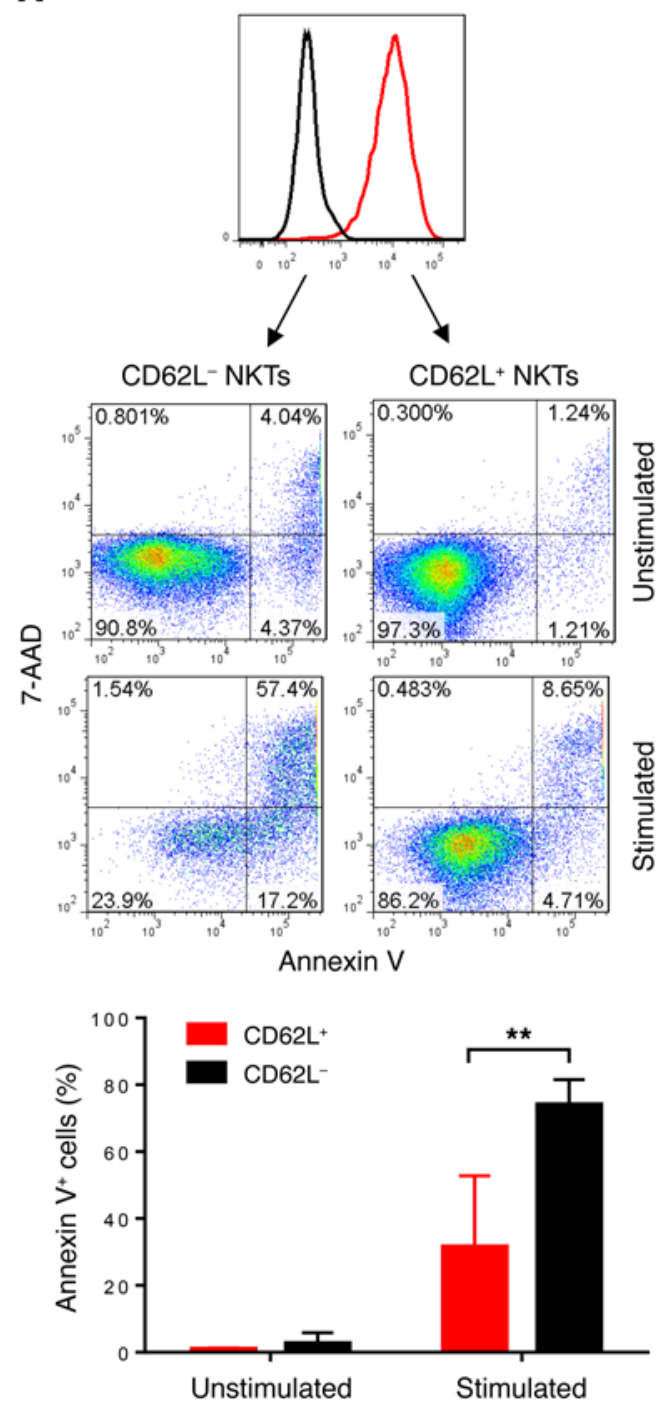

Figure 3. CD62L ${ }^{+}$NKTs have superior survival and proliferative capacity upon secondary stimulation. (A) CFSE-labeled NKTs were magnetically sorted into CD62L+ and CD62 $\mathrm{L}^{-}$subsets as confirmed by post-sorting FACS (top plot) and stimulated with irradiated $\alpha$ GalCer-pulsed APCs. On day 3 after stimulation, staining for annexin $V$ and 7-AAD was analyzed in NKTs by FACS after gating on CFSE ${ }^{+}$events. Results are from a representative of 5 donors tested (middle panel). The corresponding bar graph (bottom panel) shows mean \pm SD of percent annexin $\mathrm{V}^{+}$NKTs on day $3(n=5)$. (B) Cell proliferation was assessed on day 6 after stimulation as measured by CFSE dilution. Results are from a representative of 5 donors tested (top panel) and mean \pm SD of CFSE MFI for all donors ( $n=5$, bottom panel). (C) Total cell counts were performed at the indicated time intervals after NKT stimulation. Shown is mean \pm SD $(n=3)$ viable cells for a representative donor (top panel) or fold change for each of 5 donors tested on day 6 after stimulation. ${ }^{* *} P<0.01,{ }^{* *} P<0.001$, paired $t$ test.
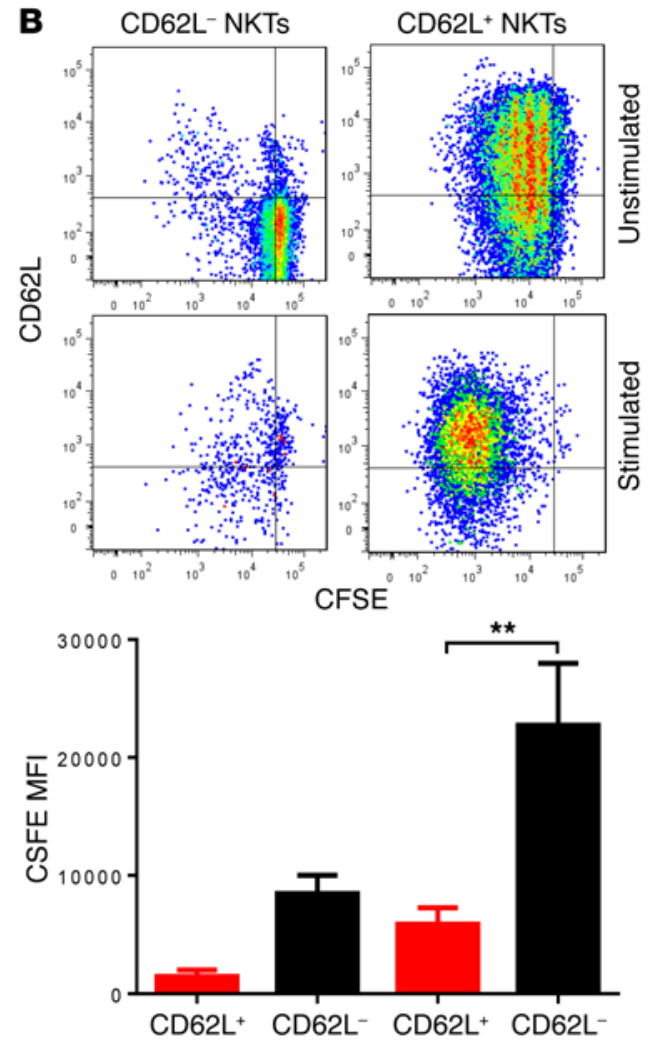

C
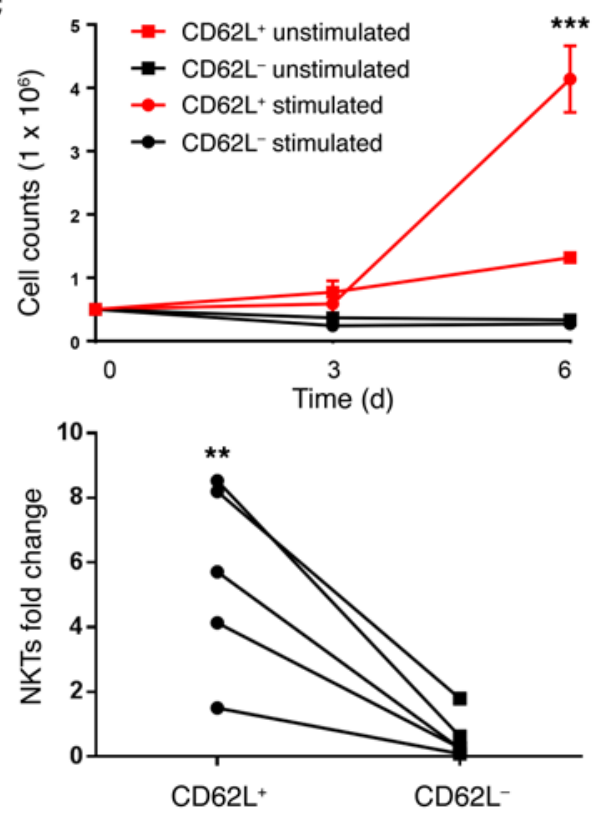

only CD62L $\mathrm{L}^{+}$CAR-NKTs induced sustained tumor regression, with 7 of 9 treated animals alive, 5 of which were tumor-free, for at least 3 months. In contrast, all 10 mice treated with $\mathrm{CD} 62 \mathrm{~L}$ CAR-NKTs succumbed to tumor progression $(P<0.001$, Figure 4 , $\mathrm{C}$ and $\mathrm{D})$. Thus, $\mathrm{CD} 62 \mathrm{~L}^{+} \mathrm{NKTs}$ have extended in vivo persistence and superior therapeutic potential compared with CD62 $\mathrm{L}^{-}$NKTs.

Costimulation maintains $C D 62 L^{+}$NKTs and prevents exhaustion. There is growing evidence that costimulation plays a critical role in the activation, survival, and expansion of NKTs (40).
While resting NKTs express CD28 (Supplemental Figure 1C), they express little or no late costimulatory receptors such as 4-1BB and OX40 (Supplemental Figure 4A). However, stimulation of NKTs with $\alpha$ GalCer-pulsed autologous peripheral blood mononuclear cells (PBMCs) resulted in rapid induction of 4-1BB in all NKTs and OX40 in CD4 $4^{+}$NKTs (Supplemental Figure 4A). Because CD62L is transiently downregulated within the first $24-48$ hours after TCR-stimulation (data not shown), we analyzed the kinetics of 4-1BB and OX40 expression in CD62 $\mathrm{L}^{+}$and CD62 $\mathrm{L}^{-}$NKTs that 
A

Days

0

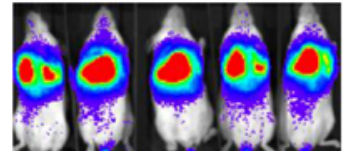

2

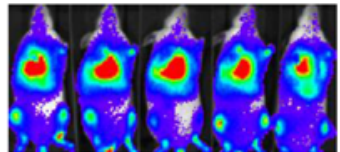

4

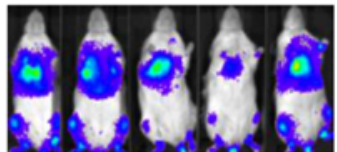

10

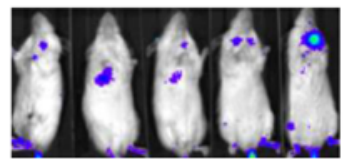

CD62L-NKTs
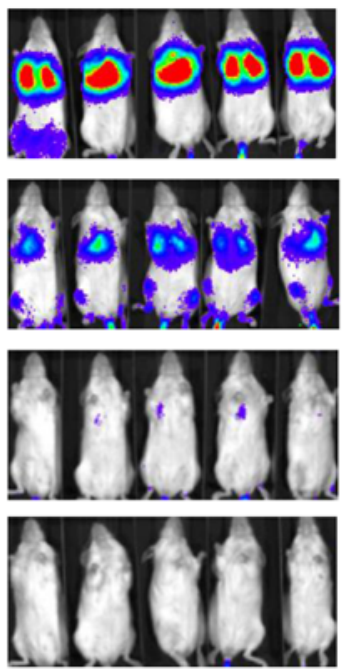

Luminescence

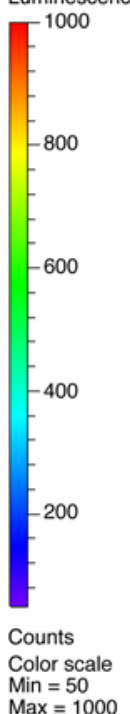

$\operatorname{Min}=50$
$\operatorname{Max}=1000$

CD62L + NKTs
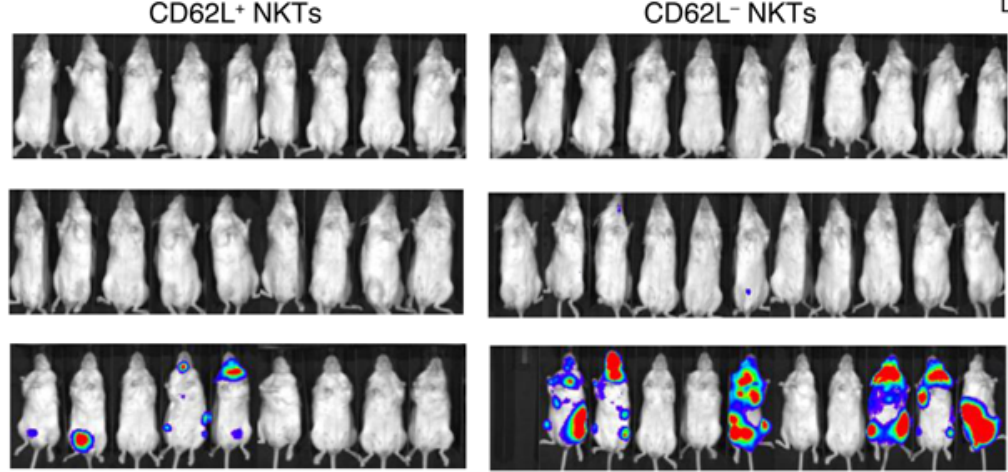

49
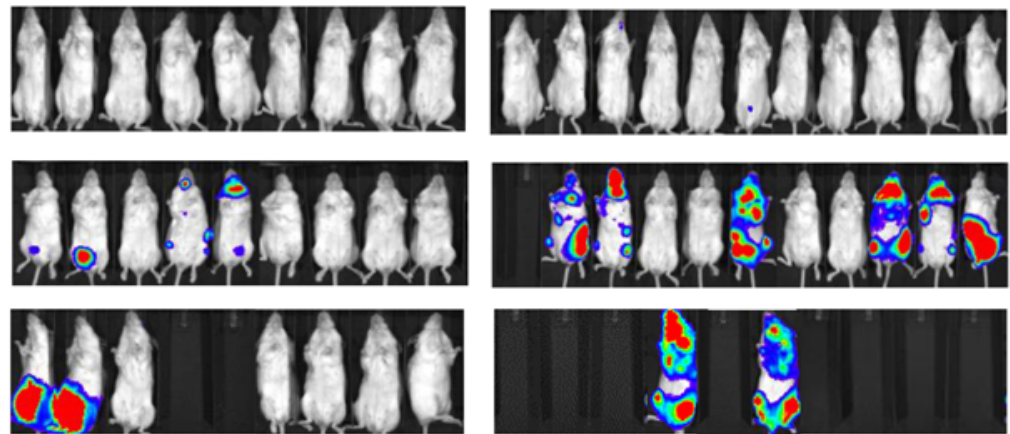

Counts

Color scale $\operatorname{Min}=200$ $\operatorname{Max}=2000$

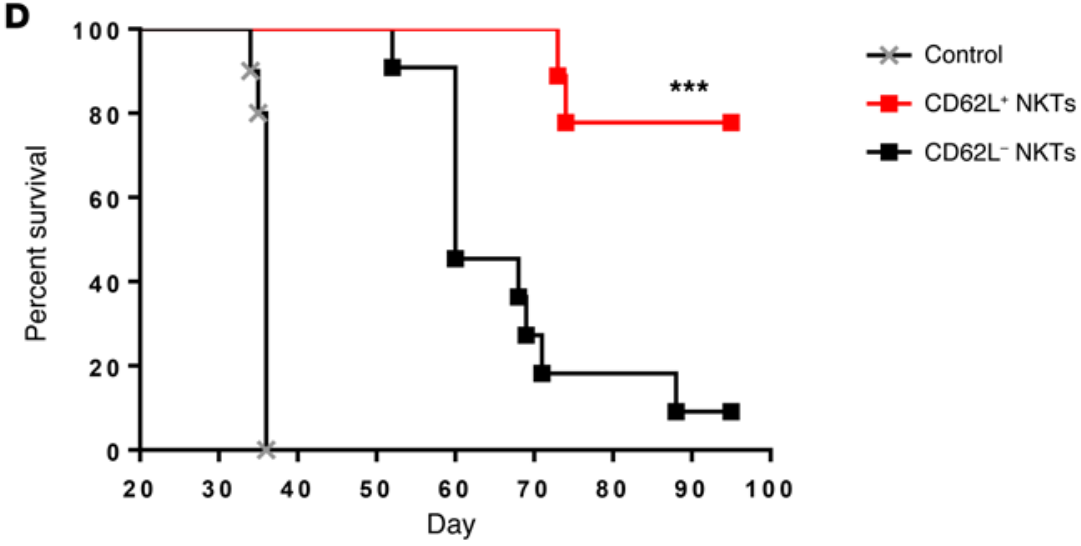

Figure 4. CD62L+ NKTs have superior in vivo persistence and antitumor activity. (A) Luciferase-transduced NKTs were sorted into CD62 $\mathrm{L}^{+}$and $\mathrm{CD}^{+} \mathrm{L} \mathrm{L}^{-}$ subsets and injected to NSG mice. NKT in vivo persistence was monitored with bioluminescence imaging. Data are from 1 of 2 independent experiments, 5 mice per group. (B) Mean \pm SD of bioluminescence photon count on indicated days after injection of CD62L' or CD62L- NKTs. Data are from the same experiment shown in $\mathbf{A}\left(P=0.008\right.$, paired $t$ test). (C) Each mouse received i.v. injection of $2 \times 10^{5}$ luciferase-transduced Daudi lymphoma cells (day 0 ) followed by (day 4) i.v. injection of $10^{7}$ CAR.CD19-transduced NKTs with IL-2 (1,000 U/mouse) or PBS as a control. Tumor growth was monitored using bioluminescent imaging once per week (10 mice per group). (D) Survival probability was analyzed by the Kaplan-Meier method (10 mice per group). The differences in survival were then compared using the log-rank test. ${ }^{* *} P<0.001$. 
A
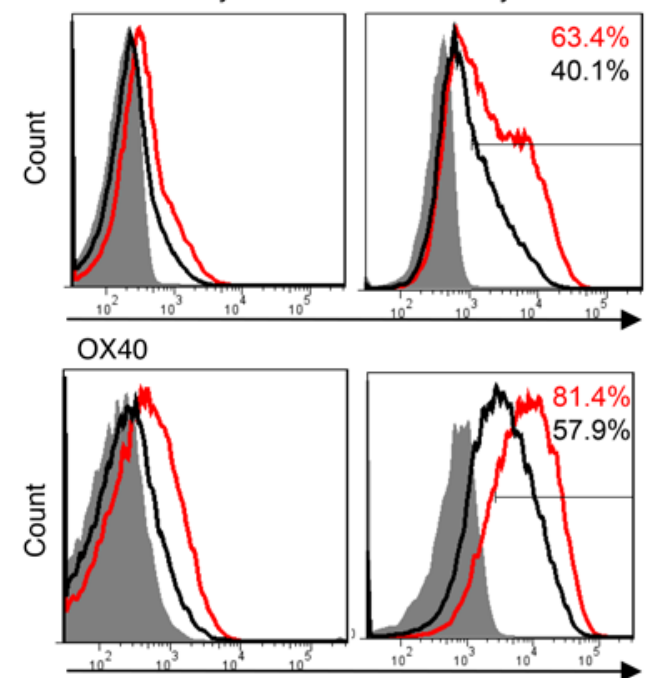

4-1BB

Day 3

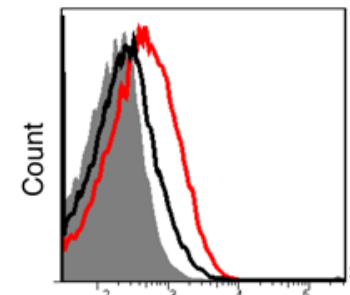

C

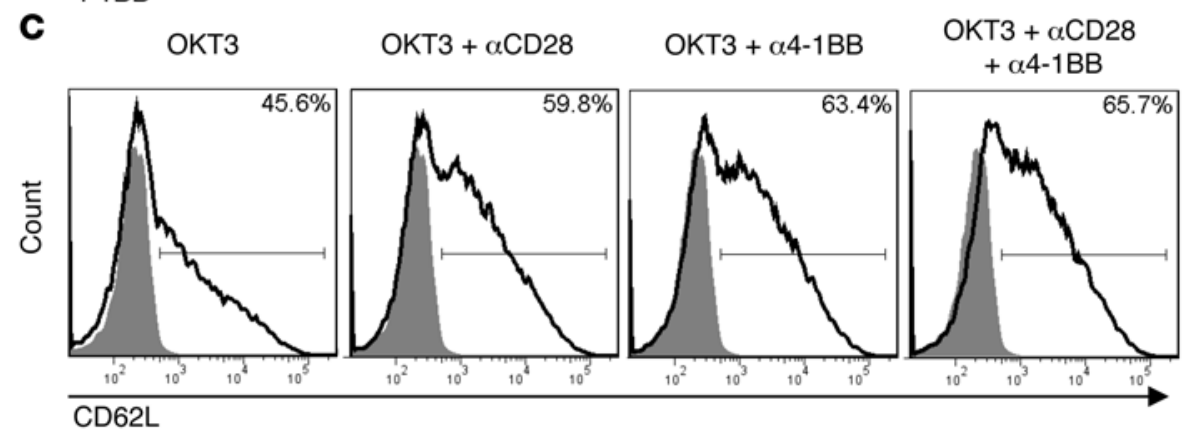

B

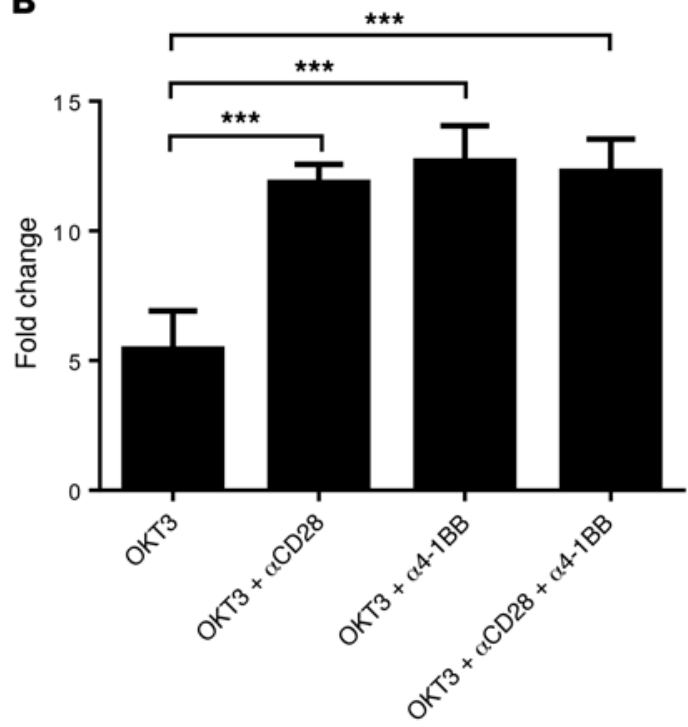

D
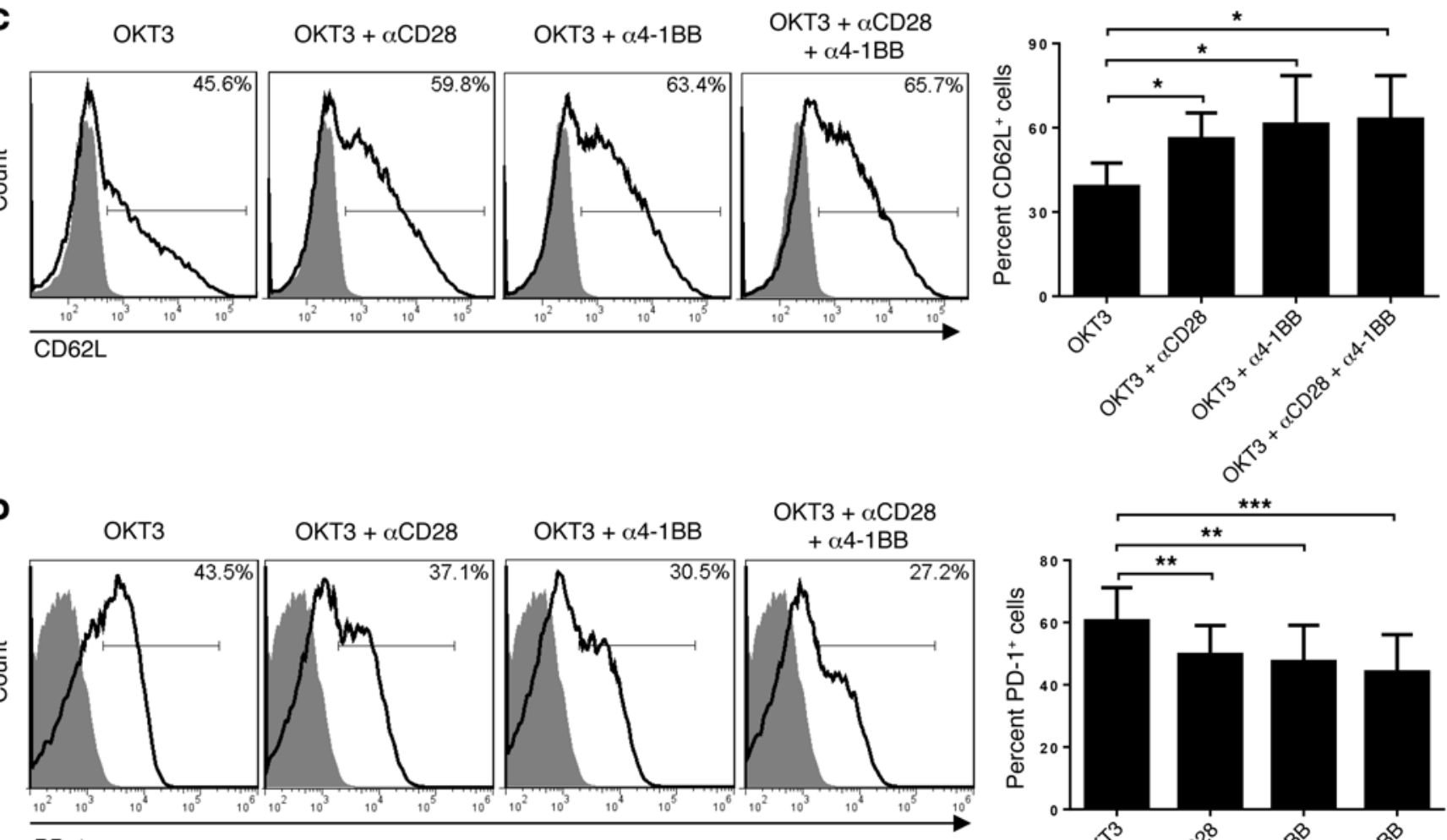

PD-1

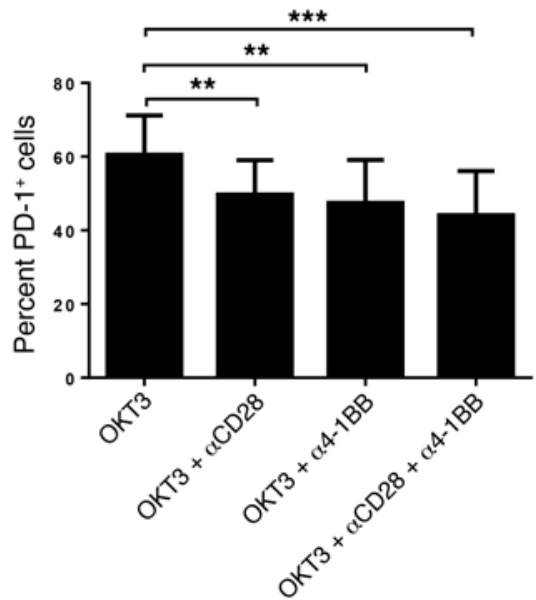

Figure 5. Costimulation maintains CD62L' NKTs and prevents exhaustion. (A) NKTs were sorted into CD62 $\mathrm{L}^{+}$and $\mathrm{CD} 62 \mathrm{~L}^{-}$subsets and stimulated and examined for expression of 4-1BB and OX40 by FACS before and 3 days after stimulation with $\alpha$ GalCer. Shown are plots from a representative of 4 donors. (B) CD62L' NKTs were stimulated on plates coated with the indicated agonistic mAbs. Shown is mean $\pm \operatorname{SD}(n=4)$ of fold change in absolute NKT number on day 7 after stimulation compared with day 0. (C) CD62 L+ NKTs were stimulated as in B and analyzed for CD62L expression (black) versus isotype control (gray) on day 7. Shown are representative overlay histograms (left panel) and mean \pm SD of percent CD62L $\mathrm{L}^{+}$cells, $n=4$. (D) CD62 $\mathrm{L}^{+}$NKTs were stimulated as in $\mathbf{B}$ and analyzed for the expression of PD-1 (black) versus isotype control (gray) on day 12. Shown are representative overlay histograms (left panel) and mean \pm SD of percent PD-1+ cells. ${ }^{*} P<0.05$, ${ }^{* *} P<0.01$, ${ }^{* *} P<0.001,1$-way ANOVA. 


\section{Table 1. Phenotype of aAPC clones $^{A}$}

\begin{tabular}{|c|c|}
\hline Clone name & Phenotype \\
\hline 22 & $\mathrm{CD} \mathrm{d}^{+}$ \\
\hline$A-2-1$ & $\mathrm{CD}^{+} \mathrm{d}^{+} \mathrm{CD} 6^{+}$ \\
\hline$A-6-2$ & $\mathrm{CD} \mathrm{d}^{+} \mathrm{CD} 6^{+} 4-1 \mathrm{BBL} \mathrm{L}^{+}$ \\
\hline$B-6-1$ & CD1d $\mathrm{d}^{+} \mathrm{CD} 86^{+} 0 \mathrm{X} 40 \mathrm{~L}^{+}$ \\
\hline B-8-2 & $\mathrm{CD} \mathrm{d}^{+} \mathrm{CD}^{2} 6^{+} 4-1 \mathrm{BBL}^{+} 0 \mathrm{OX} 40 \mathrm{~L}^{+}$ \\
\hline
\end{tabular}

were sorted before stimulation. We found that $71.13 \% \pm 18.66 \%$ and $51.98 \% \pm 18.83 \%$ of $\mathrm{CD}^{2} 2 \mathrm{~L}^{+}$and $\mathrm{CD} 62 \mathrm{~L}^{-} \mathrm{NKTs}$, respectively, upregulated OX40 within 72 hours after stimulation $(P=0.0072$, Figure 5A). Similarly, stimulated CD62 $\mathrm{L}^{+}$NKTs expressed a higher level of 4-1BB compared with CD62 $\mathrm{L}^{-}$NKTs $(P=0.011$, Figure 5A). OX40 was preferentially upregulated in the $\mathrm{CD} 4^{+}$subset of either $\mathrm{CD}^{2} \mathrm{~L}^{+}$or $\mathrm{CD} 6 \mathrm{~L}^{-} \mathrm{NKTs}$, whereas $4-1 \mathrm{BB}$ was upregulated in all NKTs (Supplemental Figure 4B).

Next, we asked whether costimulation could counteract exhaustion of in vitro expanded NKTs. CD62L $\mathrm{L}^{+}$sorted NKTs were stimulated on plates coated with an anti-CD3 agonistic $\mathrm{mAb}, \mathrm{OKT} 3$, alone or in combination with $\mathrm{mAbs}$ for CD28, 4-1BB, or both. OX40 was not tested in these settings because we could not obtain an anti-OX40 mAb with an agonistic activity. First, we found that costimulation with either anti-CD28, anti-4-1BB $\mathrm{mAb}$, or both increased the number of NKTs generated in culture within 7 days compared with stimulation with anti-CD3 alone at $20 \mathrm{ng} / \mathrm{ml}(P<0.001$, Figure $5 \mathrm{~B})$. In the absence of costimulation, increasing the concentration of OKT3 from $20 \mathrm{ng} / \mathrm{ml}$ to $1 \mu \mathrm{g} / \mathrm{ml}$ did not affect the number of NKTs generated, while costimulation was effective in increasing NKT number only when it was combined with the lower concentration of OKT3 (Supplemental Figure 5). Importantly, on day 7 after stimulation with OKT3 alone less than half the NKTs were positive for CD62L. The addition of anti-CD28, anti-4-1BB, or anti-CD28 with anti-4-1BB mAbs resulted in the retention of CD62L expression on $58 \% \pm 7.1 \%(P=$ $0.026), 73 \% \pm 9.2 \%(P=0.0036)$, and $73 \% \pm 6.1 \%(P=0.0002)$ of NKTs, respectively (Figure $5 \mathrm{C}$ ). Coordinately with the retention of CD62L expression, NKTs provided with costimulatory signals expressed significantly less PD-1 ( $P<0.05$, Figure 5D). Therefore, engagement of costimulatory receptors during antigenic stimulation supports CD62L expression in proliferating NKTs and prevents their exhaustion.

Generation of $H L A^{\text {null }}$ CD1d-expressing aAPCs with costimulatory ligands. We hypothesized that, given the observed protective effect of costimulation on TCR-activated NKTs, the optimal combination of costimulatory ligands could enable the generation of an effective aAPC for clinical-scale expansion of NKTs with high
A

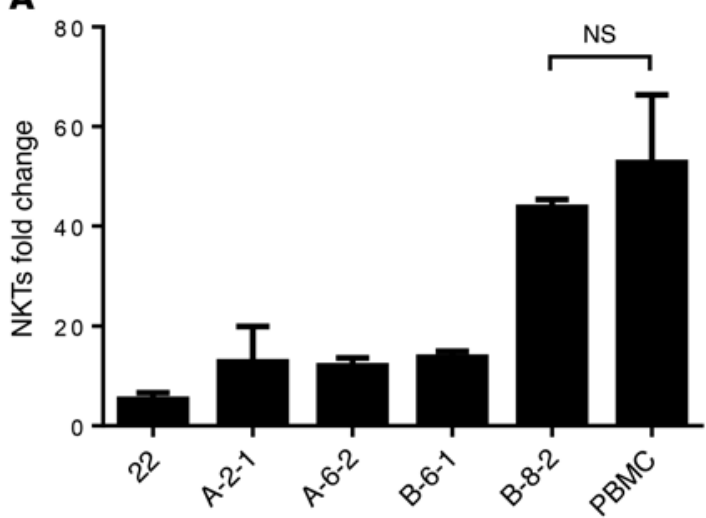

B

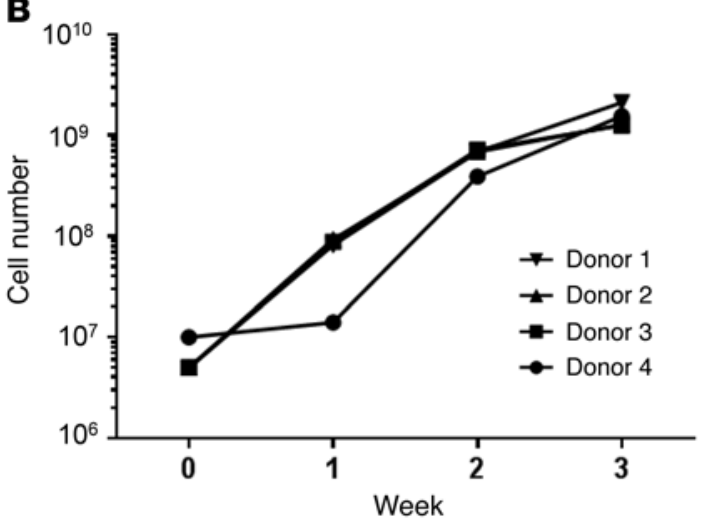

C

Donor 1

Donor 2
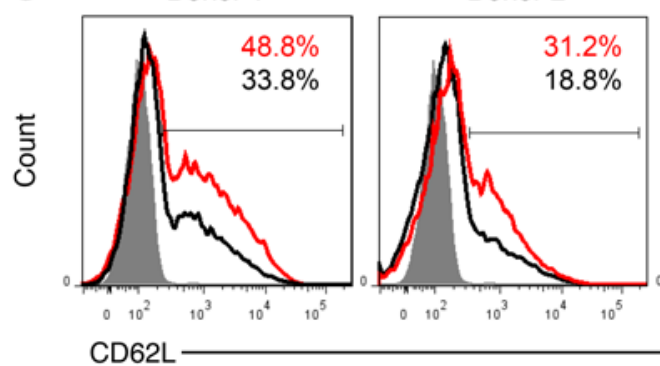

Donor 3

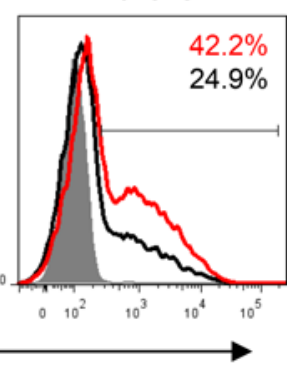

Figure 6. Generation of functional characterization of costimulatory aAPCs for NKT expansion. (A) CD1d ${ }^{+} H L A^{\text {null }}$ K562 clones were transduced with the costimulatory molecules, indicated in Table 1, irradiated, and pulsed with $\alpha$ GalCer to stimulate NKTs. NKTs were counted on day 12 after stimulation. Shown is mean $\pm \mathrm{SD}(n=3)$ of fold increase in NKT absolute number compared with day 0 for each aAPC clone from a representative of 8 experiments performed in triplicates. All groups were compared with the group in which NKTs were stimulated with autologous PBMCs, 1-way ANOVA. (B) After primary expansion with $\alpha$ GalCer-pulsed autologous PBMCs, NKTs from 4 donors were restimulated with $\alpha$ GalCer-pulsed B-8-2 clone followed by culture with IL-2. The absolute count of NKTs was performed at the indicated time intervals $(n=4)$. (C) After primary expansion with $\alpha$ GalCer-pulsed autologous PBMCs, NKTs were restimulated with autologous PBMCs or with B-8-2. CD62L expression was analyzed by FACS on day 12 using NKTs from 3 donors. $P<0.001$, paired $t$ test. 

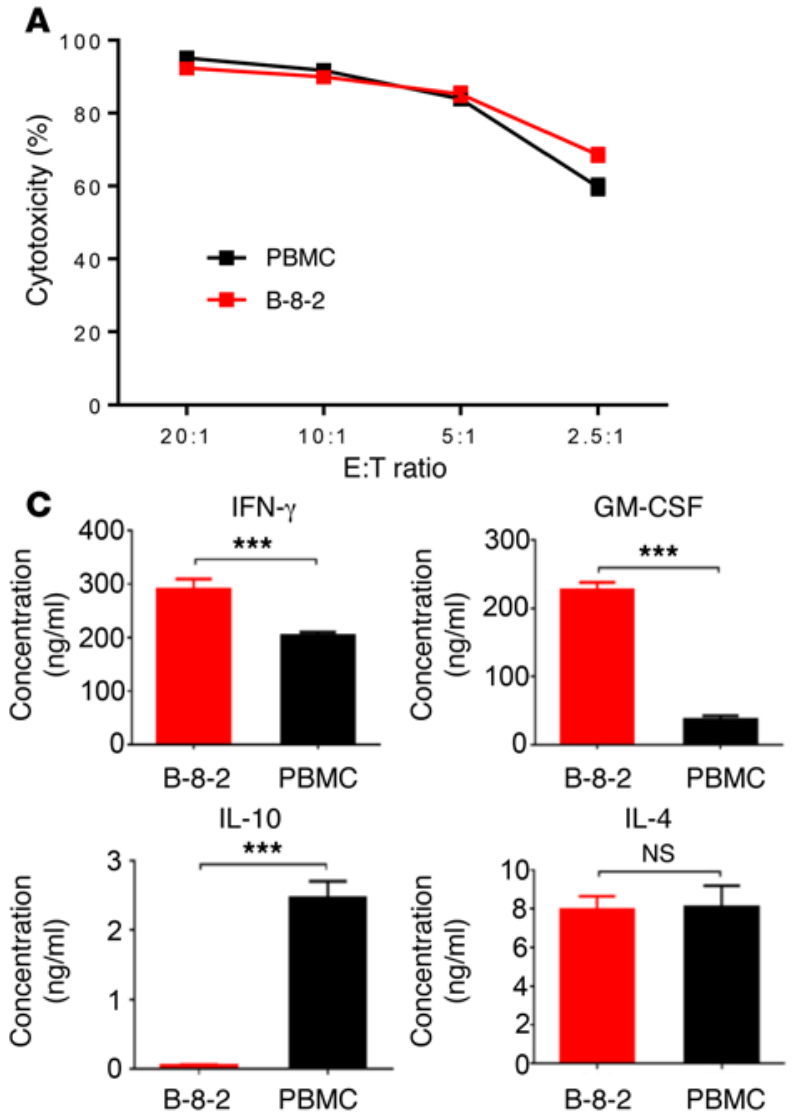

therapeutic potential. To test this hypothesis, we rendered $\mathrm{K} 562$ cells HLA $^{\text {null }}$ by eliminating the $H L A-C w 3$ gene (the only $H L A$ allele) using an HLA-C-specific zinc finger nuclease (ZFN) followed by transduction with $C D 1 d, C D 86,4-1 B B L$, and $O X 4 O L$ cDNA. HLA ${ }^{\text {null }} C D 1 d^{+}$clones expressing 1,2 , or all 3 costimulatory ligands were generated after single-cell FACS sorting, producing a total of 161 clones (Supplemental Figure 6A, and Methods section). Because the level of cell surface expression of the transduced ligands varied, we first compared the aAPC activity of clones within each subgroup. We then compared the best performers from each subgroup to one another (Table 1). Figure 6A demonstrates that clone B-8-2 with the phenotype HLA ${ }^{\text {null }} C D 1 d^{\text {med }}$ $\mathrm{CD} 6^{\text {high }} 4-1 \mathrm{BBL}^{\text {med }} \mathrm{OX} 40 \mathrm{~L}^{\text {high }}$ (Supplemental Figure 6B) consistently induced the highest rate of NKT expansion compared with other clones $(P<0.001)$, producing numbers of NKTs similar to the numbers produced by autologous PBMCs $(P>0.05$, Figure 6A). Next, we tested whether the B-8-2 clone was capable of propagating NKTs to clinically sufficient numbers. Figure $6 \mathrm{~B}$ demonstrates that 1 round of restimulation with $\mathrm{B}-8-2$ produced $272.32 \pm 111$-fold expansion of NKTs from 4 donors (range from 156 to 424 ) within 3 weeks, similar to the NKT numbers generated after stimulation with autologous PBMCs (18). However, NKTs expanded with B-8-2 contained significantly higher frequencies of CD62 $\mathrm{L}^{+}$cells compared with PBMC-expanded NKTs $(P=0.0089$, Figure 6C). Therefore, we selected B-8-2, an aAPC clone that coexpressed 3 costimulatory ligands and produced the highest rate of NKT in vitro expansion with the maximal preservation of CD62L expression.

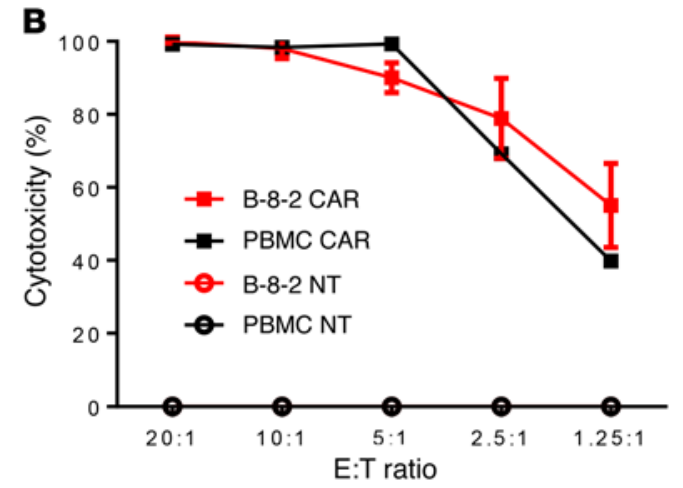

\begin{abstract}
Figure 7. Functional characterization of NKTs expanded with B-8-2. (A) Luciferase-transduced CD1d+ DAOY cells were pulsed with $\alpha$ GalCer overnight followed by coculture with NKTs that were expanded after restimulation with PBMCs or B-8-2 aAPCs. Cytotoxicity was calculated after 4 hours by measurement of luminescence intensity with a plate reader. Shown is a representative of 3 experiments in triplicates, mean $\pm S D(n=3)$. (B) NKTs were transduced with a CD19-specific CAR followed by expansion of transduced (CAR) or nontransduced (NT) NKTs with PBMC or B-8-2 restimulation. The cytotoxicity against luciferase-transduced Raji cells was analyzed as in $\mathbf{A}$. Shown is a representative of 3 experiments in triplicates, mean \pm SD $(n=3)$. (C) NKTs were expanded after restimulation with PBMCs or B-8-2 and on day 12 were stimulated with the corresponding APCs pulsed with $\alpha$ GalCer. The concentrations of IFN- $\gamma$ and IL-4 were measured in 24 -hour supernatants by Luminex assay. Shown is a representative of 3 experiments in triplicates, mean \pm SD $(n=3)$. ${ }^{* *} P<0.001$, unpaired $t$ test.
\end{abstract}

Functional properties of NKTs expanded with aAPCs. Numerous studies have demonstrated that the therapeutic potential of NKTs largely relies on their potent effector functions including cytotoxicity and cytokine production (11). To examine the effect of aAPCs on the functionality of NKTs, we expanded NKTs using PBMCs or B-8-2 and compared resultant NKTs using in vitro cytotoxicity and cytokine quantification assays. First, we tested direct NKT cytotoxicity against $\mathrm{CD} 1 \mathrm{~d}^{+} \mathrm{DAOY}$ medulloblastoma cells (41). Figure 7A demonstrates that NKTs expanded with B-8-2 and PBMCs mediated equally potent cytotoxicity against DAOY cells, killing 90\% of tumor cells at a 5:1 effector-to-target ratio. We then transduced NKTs that were expanded with B-8-2 or PBMCs with CAR.CD19 (Supplemental Figure 7) and found that both CAR-NKT preparations were equally cytotoxic against CD19+ Raji lymphoma cells, which were not killed by the parental NKTs (Figure 7B). Next, we stimulated NKTs with $\alpha$ GalCer presented by the corresponding APCs used for their expansion and measured production of Th1/Th2 cytokines using a multiplex Luminex assay. Figure 7C demonstrates that, compared with NKTs expanded and restimulated with PBMCs, those expanded and restimulated with B-8-2 produced significantly higher amounts of IFN- $\gamma$ and GM-CSF, equal amounts of IL-4, and less IL-10 $(P<0.001)$. We also performed NKT stimulation with an agonistic $6 \mathrm{~B} 11 \mathrm{mAb}$, which binds to the CDR3 region of their TCR (42), and found that while IL-10 in PBMC-stimulated NKTs was largely derived from the PBMCs, B-8-2-expanded NKTs still produced larger amounts of IFN- $\gamma$ and GM-CSF than those expanded with PBMCs (data not shown). Therefore, B-8-2- 
A Days PBMC-NT

0

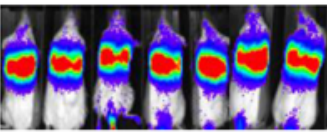

2
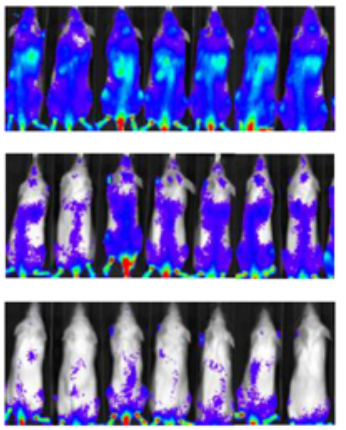

9

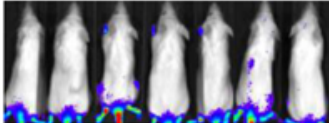

11

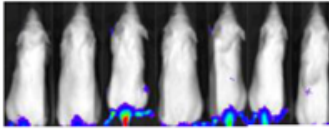

B

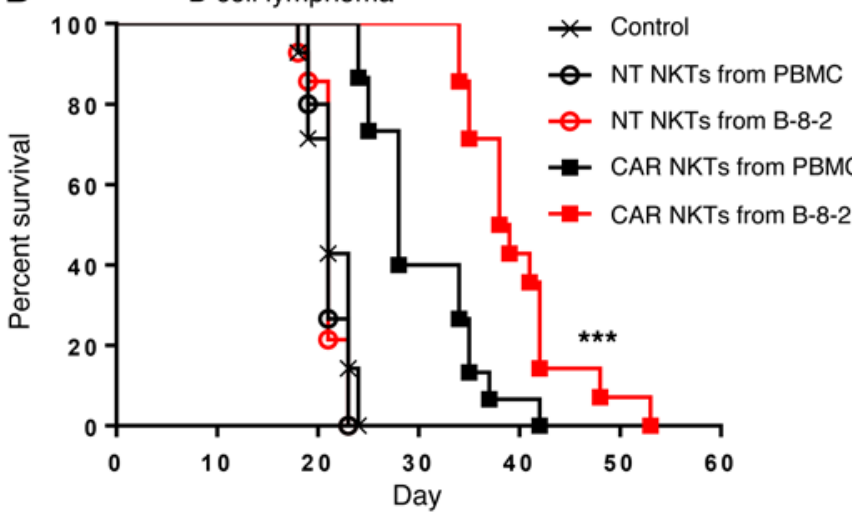

PBMC-CAR
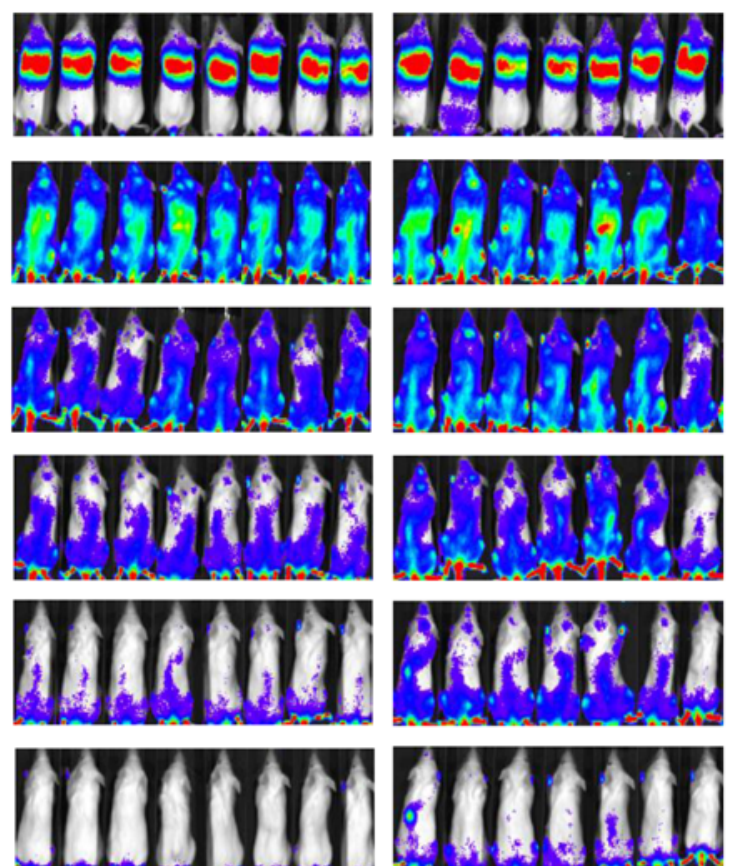

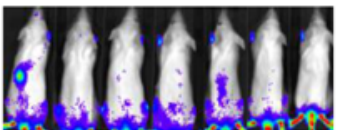

C

Neuroblastoma

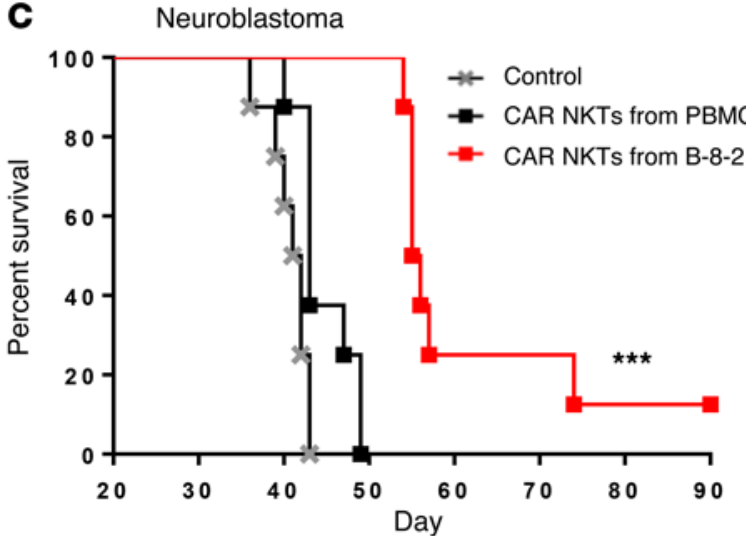

B-8-2-CAR

Luminescence
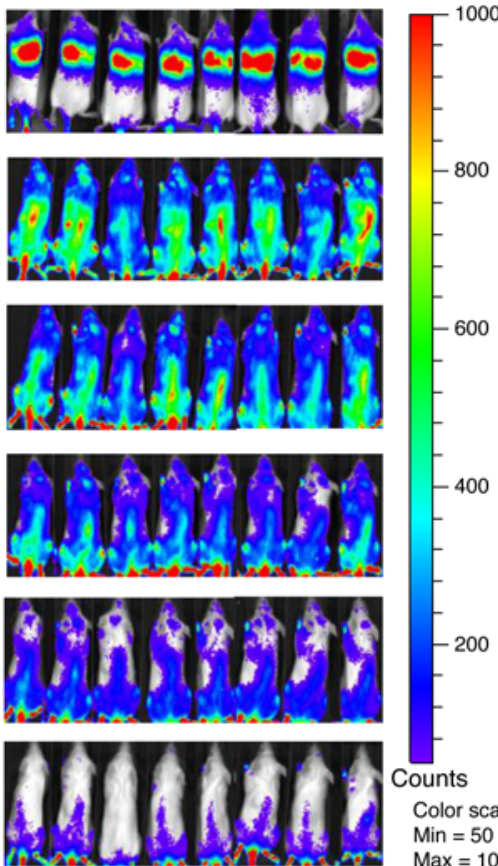

Counts

Color scale $\operatorname{Min}=50$ $\operatorname{Max}=1000$

Figure 8. aAPC-expanded NKTs have superior in vivo persistence and antitumor activity. (A) NKTs were transduced with a CD19-specific CAR followed by expansion of transduced (CAR) or nontransduced (NT) NKTs with PBMC or B-8-2 restimulation. Four groups of mice then received i.v. injection of $2 \times 10^{5}$ Raji cells (day 0) followed by (day 3) i.v. injection of the respective NKT preparations (107 cells per mouse) with IL-2 (1,000 U/mouse). NKT persistence was monitored by bioluminescent imaging on the indicated days. Bioluminescent images of 7 mice per group are shown from 1 of 2 independent experiments. (B) Mice received i.v. injection of $2 \times 10^{5}$ luciferase-transduced Raji cells (day 0) followed by (day 2) i.v. injection of $10^{7}$ NT or CAR.CD19 NKTs with IL-2 $\left(1,000 \mathrm{U} /\right.$ mouse). (C) Mice received i.v. injection of $10^{6}$ luciferase-transduced CHLA-255 cells (day 0) followed by (day 4) i.v. injection of $10^{7}$ NT or CAR.GD2 NKTs with IL-2 (1,000 U/mouse). Tumor growth was monitored using bioluminescent imaging once per week. Survival plot from 2 combined experiments for the Raji model (15 mice per group) and 1 experiment with the CHLA-255 model (8 mice per group). Data were analyzed by the Kaplan-Meier method. The differences in survival were then compared using the log-rank test. ${ }^{* *} P<0.001$.

propagated NKTs possess high levels of CD1d-dependent and CAR-redirected cytotoxicity and a Th1-biased cytokine profile.

aAPC-expanded NKTs have superior in vivo persistence and therapeutic activity. Accumulating evidence indicates that the clinical efficacy of CAR-T cell therapy against B cell malignancies critically depends on the in vivo persistence of transferred cells (43$46)$, which is influenced by the type of costimulation $(44,47)$ and phenotypic composition of CAR-T cells (48). Since we observed that the expansion of NKTs with B-8-2 affects their phenotypic composition by increasing the content of $\mathrm{CD} 62 \mathrm{~L}^{+}$cells, we compared the in vivo persistence of NKTs expanded with PBMCs and B-8-2. NKTs were transduced with luciferase alone or with CAR.
CD19 followed by adoptive transfer to NSG mice that were preimplanted with Raji lymphoma cells. Figure 8A demonstrates that CAR expression had little effect on NKT persistence, but NKTs propagated with B-8-2 persisted significantly longer than those propagated with PBMCs regardless of CAR expression $(P<0.001)$. Importantly, B-8-2-expanded CAR.CD19 NKTs significantly prolonged animal survival compared with PBMC-expanded CAR. CD19 NKTs in 2 B cell lymphoma models $(P<0.001$, Figure 8B and Supplemental Figure 8). To test whether the observed therapeutic superiority of NKTs that we had expanded with our engineered aAPCs also applies to their use against other types of cancer and target antigens, we used a metastatic model of neuro- 
blastoma with a multi-drug-resistant CHLA-255 human neuroblastoma cell line (49) and NKTs expressing a GD2-specific CAR. Figure 8C demonstrates that CAR.GD2 NKTs that were expanded with aAPCs had potent antitumor activity compared with untreated control $(P<0.001)$, whereas those expanded with PBMCs were ineffective. Therefore, expansion of NKTs using B-8-2 aAPCs confers a qualitative advantage to NKTs, enabling superior in vivo persistence and antitumor activity.

\section{Discussion}

A critical gap in knowledge of human NKT biology has slowed down the development of effective NKT-based cancer immunotherapy. We found that NKT numeric ex vivo expansion and subsequent in vivo persistence - essential requirements for effective NKT-based cell and gene therapy applications - depend on the $\mathrm{CD} 2 \mathrm{~L}^{+}$subset of peripheral blood NKTs. Only CD62L ${ }^{+}$NKTs survive and proliferate in response to repeated TCR-stimulation, while CD62 $\mathrm{L}^{-}$cells undergo early exhaustion and cell death. Although continuous stimulation of NKTs is associated with the loss of CD62L expression, activation of costimulatory receptors during TCR-stimulation can counteract this process. Specifically, we experimentally determined a unique combination of CD86, 4-1BBL, and OX4OL molecules and the levels of their coexpression on the surface of an aPC that enables highly efficient clinical-scale NKT expansion with maximal preservation of CD62L expression. CAR-NKTs generated using the aAPCs developed in the course of this study demonstrated extended in vivo persistence and superior therapeutic activity against in vivo models of lymphoma and neuroblastoma.

We show that the $\mathrm{CD} 62 \mathrm{~L}^{+}$subset is responsible for the numeric expansion of NKTs upon antigenic stimulation ex vivo. The following findings support the above conclusion: (a) the fraction of $\mathrm{CD} 2 \mathrm{~L}^{+}$cells is dramatically increased after primary NKT stimulation; (b) the majority of sorted $\mathrm{CD}^{2} 2 \mathrm{~L}^{-}$cells undergo apoptosis, whereas sorted $\mathrm{CD}_{62} \mathrm{~L}^{+}$cells proliferate in response to identical stimulation; and (c) CD62 $\mathrm{L}^{-}$NKTs exhibit signs of terminal differentiation (CD161 and CD56 expression) in freshly isolated PBMCs and rapidly acquire an exhaustion phenotype upon in vitro stimulation as evidenced by marked upregulation of PD-1 and TIM-3 expression and diminished ability to produce cytokines. When similar characteristics are observed in T cell therapeutic products, they are associated with a subsequent lack of persistence or of objective responses after adoptive transfer to cancer patients $(50,51)$.

We observed that proliferating NKTs eventually downregulate CD62L expression and acquire an exhaustion phenotype during in vitro culture. This observation likely reflects the ontogeny of human peripheral NKTs, as the frequency of CD62L $\mathrm{L}^{+} \mathrm{NKT}$ is lower in adult peripheral blood compared with cord blood $(33,52)$. In 1 report, cord blood NKTs were found not to express CD62 (31). The reason for the discrepancy between that report and others and with our own results is not clear and is likely to be technical. Constantinides et al. identified a very rare naive-like population of CD1d-restricted $\mathrm{T}$ cells with a high level of CD62L in peripheral blood (53). However, these cells do not express the invariant V $\alpha 24$ chain and have a lower level of PLZF expression compared with the classical NKTs. Both CD62L $\mathrm{L}^{+}$and CD62- NKTs in this study expressed equally high levels of PLZF.
CD62L may represent a common marker of cells responsible for the long-term maintenance of peripheral NKT and T cells. Graef et al. demonstrated that $\mathrm{CD} 62 \mathrm{~L}^{+}$central memory $\mathrm{T}$ cells possess stem cell properties; they could propagate themselves while giving rise to effector-memory and effector $\mathrm{T}$ cells (36). In the most recently published study, Sommermeyer et al. demonstrated that human CAR.CD19-expressing CD8 or CD4 T cells generated from naive and central-memory subsets were more effective against Raji lymphoma xenografts compared with those generated from effector-memory subsets (38). The authors also found that combining the most potent $\mathrm{CD} 4^{+}$and $\mathrm{CD} 8^{+} \mathrm{CAR}$-expressing subsets produced synergistic antitumor activity in vivo. Activation of NKTs has been shown to lead to transactivation of NK and CD8 T cells in murine models and in human clinical trials $(6,54)$, so that the combination of CAR-NKTs with other defined subsets of CAR-expressing lymphocytes may be a useful therapeutic strategy.

$L E F 1$ and $I L 7 R A$ were among the immune-related genes that were most overexpressed in $\mathrm{CD}_{62} \mathrm{~L}^{+}$compared with CD62 $\mathrm{L}^{-} \mathrm{NKTs}$. In a recent report, Carr et al. demonstrated a unique function of LEF1 in the expansion of murine Va14-invariant (iNKT) cells during stage 2 of their thymic development via direct transcriptional activation of CD127 and $c-M Y C$ gene expression (39). Consistent with our observation of the coordinated expression of LEF1 and GATA3 in human NKTs, these investigators also found that LEF1 upregulates transcription factor GATA3, which is required for IL-4 production in iNKT2 cells as well as for dual production of IL-4 and IFN- $\gamma$ in iNKT1 cells. The latter cells closely resemble human peripheral blood NKTs, in which we found that CD62 $\mathrm{L}^{+}$cells preferentially express GATA3 and produce high levels of both cytokines. Taking the Carr et al. study with murine NKTs and our own results with human NKTs, we suggest that LEF1 plays a critical role at early stages of NKT development and controls their number and function. The high level of LEF1 expression in $\mathrm{CD} 62 \mathrm{~L}^{+}$and its loss in CD62 $\mathrm{L}^{-}$subsets of human NKTs is consistent with a model of linear progression from less differentiated CD62L $\mathrm{L}^{+}$NKTs with a preserved proliferative potential and ThO-like cytokine profile toward terminally differentiated CD62L- NKTs with diminished ability to proliferate and produce cytokines.

There is growing evidence that costimulation plays a critical role in the development, activation, and functional responses of NKTs in murine models $(40,55)$. However, little is known about the expression of costimulatory receptors in human NKTs. In this study, we focused on a set of costimulatory receptors that have pronounced prosurvival properties in human T cells: CD28, 4-1BB, and OX40 (56-58). First, we confirmed that freshly isolated human NKTs express CD28 (34) and showed that they coexpress CD27, thereby resembling the corresponding stage of memory $\mathrm{T}$ cell differentiation with preserved functional potential (35). To our knowledge, the current study is the first to characterize the baseline and post-stimulation kinetics of 4-1BB and OX40 in human NKTs. We found that both receptors are undetectable in freshly isolated NKTs but are induced following TCR-stimulation. Like T cells (59), human NKTs preferentially upregulated OX40 in the $\mathrm{CD} 4^{+}$subset. The majority of NKTs also upregulated 4-1BB, which is preferentially upregulated in the $\mathrm{CD}^{+}$subset of $\mathrm{T}$ cells (60). Importantly, we found that costimulation of individual costimulatory receptors could inhibit the loss of CD62L expression and res- 
cue NKTs from exhaustion. We also observed an additive effect on the maintenance of CD $62 \mathrm{~L}^{+} \mathrm{NKT}$ s when CD28 and 4-1BB were simultaneously activated.

Several types of aAPCs have been developed from the K562 cell line for clinical-scale numeric expansion of T and NK cells for adoptive immunotherapy applications (61-63). In the design of K562-based aAPCs for NKTs, we sought to selectively activate and expand NKTs while avoiding competition with $\mathrm{T}$ and NK cells. K562 cells express only 1 HLA allele, HLA-Cw3, which is known to activate NK cells (64). Hence, the deletion of the $H L A-C w 3$ gene and transgenic expression of CD1d in HLA ${ }^{\text {null }}$ K562 cells enabled antigen presentation to NKTs without activation of NK or conventional T cells. Importantly, the B-8-2 clone we experimentally selected with the optimal combination of costimulatory ligands (and their expression levels) demonstrated aAPC qualities for NKTs that exceeded those of the autologous PBMCs. We did, however, observe a differential effect on NKT expansion between plate-bound agonistic mAbs and the corresponding ligands for costimulatory receptors. While costimulation with 1 or $2 \mathrm{mAbs}$ for CD28 or $4-1 \mathrm{BB}$ enhanced the rate of NKT expansion with retention of CD62L, expression of ligands for 3 receptors (CD28, 4-1BB, and OX40) on K562 cells was needed to achieve such an effect. This could be explained by higher affinity of mAbs to the receptors compared with the natural ligands or expression of inhibitory molecules on K562 cells that counteract NKT activation.

B-8-2-expanded NKTs and CAR-NKTs had superior in vivo persistence, one of the critical requirements for the efficacy of adoptive cancer immunotherapy. Indeed, we found that B-8-2expanded NKTs expressing anti-CD19 and anti-GD2 CARs had superior therapeutic activity in B cell lymphoma and neuroblastoma models, respectively. In this study, we do not compare NKTs or CAR-NKTs with T or CAR-T cells because the xenogenic model in NSG mice does not allow such a comparison. It is well documented that adoptive transfer of human T or CAR-T cells to NSG mice leads to the development of xeno-graft-versus-host disease $(\mathrm{GvHD})(18,65,66)$. The latter report revealed that unless depleted from $\mathrm{CD}_{45 \mathrm{RA}^{+}}$naive cells before ex vivo expansion, human $\mathrm{T}$ cells with or without anti-CD19 CAR cause severe xenogeneic GvHD in addition to antileukemia activity. In contrast, the persistence of human NKTs or CAR-NKTs in mice is not supported by the artifact of xenoreactivity to murine tissues (18).

Despite recent successes in the immunotherapy of $\mathrm{B}$ cell leukemias and lymphomas with CAR-T cells, about half of lymphoma patients do not achieve complete responses, and the duration of the responses is not yet well established $(67,68)$. NKTs could have a particular advantage as CAR carriers for the immunotherapy of B cell malignancies, including lymphomas in the settings of allogeneic hematopoietic stem cell transplant (HSCT), because NKTs do not induce and even suppress GvHD while maintaining graft-versus-leukemia activity $(69,70)$. Moreover, a recent report demonstrated that reconstitution of NKTs in peripheral blood is associated with long-term remission in pediatric leukemia patients receiving haploidentical stem cell transplantation $(71,72)$. Hence, unlike donor-derived CAR-T cells, CAR-NKTs can be given early after HSCT when the disease burden is minimal and the curative potential of immunotherapy could be maximized. Further enhancement of CAR-NKT persistence and antitumor efficacy could be achieved by providing optimal costimulatory signals within CAR constructs such as CD28 or $4-1 \mathrm{BB}$ costimulatory endodomains that are critically important for the clinical efficacy of CAR.CD19 T cells $(19,20)$. The optimal costimulatory CAR design for NKTs is yet to be found. It will be interesting to determine the role of the CD $62 \mathrm{~L}^{+}$ subset in CAR-mediated NKT proliferation and persistence. Ultimately, the development of an effective NKT-based CAR-redirected cancer immunotherapy will likely require the coordinated use of costimulation provided by aAPCs during ex vivo expansion and CAR after adoptive transfer to patients.

\section{Methods}

Cell lines and culture conditions. The parental K562 cells were provided by C. June (University of Pennsylvania, Philadelphia, PA). Daudi, Raji, DAOY, and 293T cells were purchased from American Type Culture Collection (ATCC). The CHLA-255 cell line was previously described (49). K562, Daudi, Raji, and CHLA-255 cells were cultured in RPMI, whereas DAOY and 293T cells were maintained in Iscove's Modified Dulbecco's Medium (IMDM; Invitrogen). Both types of medium were supplemented with 10\% FBS (HyClone), 2 mM GlutaMAX-1 (Gibco-BRL).

NKT isolation, transduction, expansion, and sorting. To analyze the cord blood NKTs we used discarded cord blood units obtained from the MD Anderson Cancer Center Cord Blood Bank according to the protocols approved by the IRBs at MD Anderson Cancer Center and Baylor College of Medicine (BCM). PBMCs of healthy donors (at least 18 years old) were isolated by gradient centrifugation from buffy coats purchased from Gulf Coast Regional Blood Center. NKTs were purified by anti-iNKT microbeads (Miltenyi Biotec). The negative PBMC fraction was irradiated (40 Gy) and aliquoted. NKTs were stimulated with an aliquot of autologous PBMCs pulsed with $100 \mathrm{ng} / \mathrm{ml} \alpha \mathrm{GalCer}$ (Kyowa Hakko Kirin). Recombinant IL-2 (200 U/ml; National Cancer Institute Frederick) was added every other day in complete RPMI (HyClone RPMI 1640, 10\% heat-inactivated FBS, and 2 mM GlutaMAX). NKTs were expanded for 10 days and then restimulated with autologous PBMCs (irradiated with 40 Gy) or aAPCs (irradiated with $100 \mathrm{~Gy})$ when indicated. On day 3 after restimulation, 24-well, nontissue culture plates were coated with retronectin (Takara Bio) and, after washing, inoculated with $1 \mathrm{ml}$ of retroviral supernatant containing CAR.CD19 and spun for 60 minutes at $4,600 \mathrm{~g}$. The viral supernatant was then removed, and stimulated NKTs were added to the wells in complete media and $200 \mathrm{U} / \mathrm{ml}$ recombinant human IL-2. Cells were removed from the plate after 48 hours, washed, resuspended at the concentration $10^{6}$ cells $/ \mathrm{ml}$ in complete RPMI with $200 \mathrm{U} / \mathrm{ml} \mathrm{IL-2,} \mathrm{and}$ plated for continued expansion. NKT number was determined by trypan blue (Life Technologies) counting. When indicated, NKTs or CARNKTs were labeled with CD62L-PE mAb (GREG-56; BD Biosciences) and anti-PE microbeads (Miltenyi) followed by magnetic sorting into CD62 $\mathrm{L}^{+}$and $\mathrm{CD} 62 \mathrm{~L}^{-}$subsets according to the manufacturer's instructions. The phenotype of the sorted cells was determined by FACS.

Retroviral constructs and retrovirus production. CAR.CD19 and CAR. GD2 constructs were made as previously described $(18,73)$ and contained a single-chain variable fragment from the CD19-specific antibody FMC-63 or the GD2-specific antibody 14G2a connected via a short spacer derived from the IgG1 hinge region to the transmembrane domain derived from $\operatorname{CD} 8 \alpha$, followed by signaling endodomain sequences of 
4-1BB fused with $\zeta$ chain. Retroviral supernatants were produced by transfection of $293 \mathrm{~T}$ cells with a combination of CAR-containing plasmid, RDF plasmid encoding the RD114 envelope, and PegPam3 plasmid encoding the MoMLV gag-pol as previously described (74).

Proliferation and apoptosis assays. NKTs were labeled with CFSE (Invitrogen) and stimulated with $\alpha$ GalCer-pulsed PBMCs, aAPC clones, or plates coated with $20 \mathrm{ng} / \mathrm{ml}$ anti-CD3 (OKT3) alone or in combination with $0.5 \mu \mathrm{g} / \mathrm{ml}$ anti-CD28 (CD28.2) and/or $1.5 \mu \mathrm{g} / \mathrm{ml}$ anti-4-1BB (h41BB-M127) (BD Biosciences). Cell proliferation was examined on days 3 and 6 by measurement of CFSE dilution using flow cytometry. In addition, early and late stages of apoptosis were measured on day 3 by staining with annexin $\mathrm{V}$ and 7-AAD (BD Biosciences) followed by flow cytometry.

Multiplex cytokine quantification assay. NKTs were stimulated for 24 hours with either APCs or agonistic antibody-coated plates (clone 6B11; BD Biosciences). Supernatants were collected and analyzed with a Human Cytokine/Chemokine Immunoassay kit (Millipore) using the Luminex assay according to the manufacturer's instructions.

Flow cytometry. Immunophenotyping was performed using the following mAbs: HLA-C EMR8-5, CD1d 42.1, CD86 2331, 4-1BBL C65-485, OX40L ik-1, CD3 HIT3a, Va24-Ja18 6B11, CD4 SK3, CD62L DREG-56, CD134 ACT35, CD137 4B4-1, PD-1 EH12.1, GATA3 L50823, PLZF R17-809, CCR7 3D12, CD28 CD28.2, CD27 L128, IL-7R HIL7R-M21, CD161 DX12, CD56 B159 (BD Biosciences), LAG-3 polyclonal, TIM-3 344823 (R\&D Systems), and rabbit anti-LEF1 EP2030Y mAb (Abcam). Fluorochrome- and isotype-matching antibodies suggested by BD Biosciences or R\&D Systems were used as negative controls. The expression of CAR.CD19 on NKTs was determined using anti-Id (clone 136.20.1) CAR.CD19-specific mAb (75) and goat anti-mouse IgG (BD Biosciences). The expression of CAR.GD2 on NKTs was detected using the anti-idiotype $1 \mathrm{~A} 7 \mathrm{mAb}$, which was produced in house from murine hybridoma HB-11786 (ATCC) followed by staining with a secondary rat anti-mouse IgG1-PE mAb (BD Biosciences). The analysis was performed on an LSR-II 5-laser flow cytometer (BD Biosciences) using BD FACSDiva software version 6.0 and FlowJo 7.2.5 (Tree Star).

In vitro cytotoxicity assay. The cytotoxicity of parental and CAR. CD19 NKTs against DAOY or Raji cells was evaluated using 4-hour luciferase assay as previously described (41).

Generation of aAPC clones. We rendered K562 cells HLA ${ }^{\text {null }}$ by eliminating the $H L A-C$ gene from the K562 cell genome using an HLA-Cspecific ZFN as previously described (76). Briefly, ZFNs containing 5 or 6 fingers were designed and assembled using an established archive of prevalidated 2-finger and 1-finger modules (Sangamo BioScience). The coding sequence of HLA-C was scanned for locations at which 2 such ZFNs (designated as ZFN-L and ZFN-R) could be targeted to sites that were separated by $5 \mathrm{bp}$ and located on opposite DNA strands. The nucleotide targets for candidate ZFN pairs were then checked for divergence from other HLA coding sequences. Genes encoding the ZFNs were assembled using a PCR-based procedure and cloned into a plasmid. For the modification of K562 cells, expression vectors encoding HLA-C-targeting ZFNs (Supplemental Figure 4A) were introduced by Nucleofection (Lonza) according to the manufacturer's protocol. After electroporation, cells were immediately placed in prewarmed complete medium. HLA-C-negative clones were obtained using limiting dilution, and HLA deletion was confirmed by Surveyor nuclease assay (76). A clone of HLA ${ }^{\text {null }}$ K562 cells was then electroporated with human CD1d cDNA using Sleeping Beauty transposon/transposase system as previously described (76). A clone with an intermediate level of CD1d expression induced the highest rate of NKT proliferation and was further modified with lentiviral vectors encoding CD86, 4-1BBL, or OX40L followed by single cell sorting and clonal expansion. The generated library of clones was divided into groups based on expression of 1, 2, or 3 costimulatory molecules.

Gene expression analysis. Total RNA was collected using TRIzol reagent (Qiagen). Gene expression analysis was performed using Immunology Panel version 2 (NanoString) at BCM Genomic and RNA Profiling Core using nCounter Analysis System. Data were analyzed using nSolver 2.0 software (NanoString).

In vivo experiments. The colony of NSG mice was originally obtained from The Jackson Laboratory and maintained at BCM Animal Care facility. Tumor growth was initiated by i.v. injection of $2 \times$ $10^{5}$ luciferase-transduced Raji lymphoma cells. On day 3, mice were treated with $4 \times 10^{6}$ to $8 \times 10^{6} \mathrm{CAR}-\mathrm{NKT}$ s followed by i.p. injection of IL-2 (1,000 U/mouse) every 3 days. Tumor growth was assessed twice per week by bioluminescent imaging (Small Animal Imaging core facility, Texas Children's Hospital). For the in vivo persistence experiments, NKTs were cotransduced with CAR.CD19 and luciferase using retroviral constructs, i.v. injected to tumor-free or tumor-bearing mice, and monitored using bioluminescent imaging twice per week. Animal experiments were performed according to IACUC-approved protocols.

Statistics. For in vitro and in vivo experiments, we used 2-sided, paired Student's $t$ test to evaluate continuous variable of 2 groups, and 1-way ANOVA with post-test Bonferroni to evaluate continuous variables of more than 2 groups. Survival was analyzed by the KaplanMeier method and the log-rank (Mantel-Cox) test to compare pairs of groups. Statistics were computed using GraphPad Prism 5.0 (GraphPad Software). Differences were considered significant when the $P$ value was less than 0.05 .

Study approval. The cord blood units were obtained from MD Anderson Cancer Center Cord Blood Bank according to the protocols approved by the IRBs at MD Anderson Cancer Center (H-16320) and Baylor College of Medicine (H-20911). Written informed consent was received from all participating women prior to inclusion in the study under the protocol H-16320. Cord blood units not suitable for clinical use (usually because of low cell counts) were either discarded or used for research purposes under the protocol H-20911 at BCM.

Animal experiments were performed according to the protocol AN-5194 approved by the IACUCs of BCM.

\section{Author contributions}

$\mathrm{NKT}$ isolation, expansion, transduction, and in vitro functional testing were done by GT, ANC, AH, DL, and XX. In vivo experiments were performed by GT, ANC, EM, and LG. Design and genetic engineering of K562-based aAPCs were performed by BJ, HT, GT, LJC, and LSM. Functional testing of aAPC clones and selection of B-8-2 clone was accomplished by GT. CAR constructs were made by GD. Data analysis including statistical analysis was performed by GT, ANC, LSM, and QM. The manuscript was written by GT and LSM and edited by GD and LJC.

\section{Acknowledgments}

We thank Malcolm Brenner and Catherine Gillespie (Center for Cell and Gene Therapy, BCM, Houston, TX) for helpful discussions and scientific editing. This work was supported by grants 
from the National Institutes of Health (RO1 CA116548 to L.S. Metelitsa; R01 CA142636 to G. Dotti; P50 CA126752 to L.S. Metelitsa and G. Dotti), Cancer Prevention and Research Institute of Texas (RP130588 to L.S. Metelitsa), Alex's Lemonade Stand Foundation for Childhood Cancer (to L.S. Metelitsa), and Cookies for Kids' Cancer Foundation (to L.S. Metelitsa).

Address correspondence to: Leonid S. Metelitsa, Department of Pediatrics, Baylor College of Medicine, 1102 Bates Avenue,
C.1760.06, Houston, Texas 77030, USA. Phone: 832.824.4395;

E-mail:1smeteli@txch.org.

L.J. Cooper's present address is: Ziopharm Oncology Inc., Boston, Massachusetts, USA.

G. Dotti's present address is: Department of Microbiology and Immunology, University of North Carolina, Chapel Hill, North Carolina, USA.
1. Porcelli S, Yockey CE, Brenner MB, Balk SP. Analysis of $\mathrm{T}$ cell antigen receptor (TCR) expression by human peripheral blood CD $4-8-\alpha / \beta$ T cells demonstrates preferential use of several $\mathrm{V}$ beta genes and an invariant TCR $\alpha$ chain. J Exp Med. 1993;178(1):1-16.

2. Lantz O, Bendelac A. An invariant T cell receptor $\alpha$ chain is used by a unique subset of major histocompatibility complex class I-specific $\mathrm{CD}^{+}$and CD4-8- T cells in mice and humans. J Exp Med. 1994;180(3):1097-1106.

3. Bendelac A, Lantz O, Quimby ME, Yewdell JW, Bennink JR, Brutkiewicz RR. CD1 recognition by mouse $\mathrm{NK}^{+} \mathrm{T}$ lymphocytes. Science. 1995;268(5212):863-865.

4. Kim EY, Lynch L, Brennan PJ, Cohen NR, Brenner MB. The transcriptional programs of iNKT cells. Semin Immunol. 2015;27(1):26-32.

5. McEwen-Smith RM, Salio M, Cerundolo V. The regulatory role of invariant NKT cells in tumor immunity. Cancer Immunol Res. 2015;3(5):425-435.

6. Dhodapkar MV. Harnessing human CD1d restricted T cells for tumor immunity: progress and challenges. Front Biosci (Landmark Ed). 2009;14:796-807.

7. Exley MA, Nakayama T. NKT-cell-based immunotherapies in clinical trials. Clin Immunol. 2011;140(2):117-118.

8. Motohashi S, Okamoto Y, Yoshino I, Nakayama T. Anti-tumor immune responses induced by iNKT cell-based immunotherapy for lung cancer and head and neck cancer. Clin Immunol. 2011;140(2):167-176.

9. Yamasaki K, et al. Induction of NKT cell-specific immune responses in cancer tissues after NKT cell-targeted adoptive immunotherapy. Clin. 2011;138(3):255-265.

10. Taniguchi M, Harada M, Dashtsoodol N, Kojo S. Discovery of NKT cells and development of NKT cell-targeted anti-tumor immunotherapy. Proc Jpn Acad Ser B Phys Biol Sci. 2015;91(7):292-304.

11. Metelitsa LS. Anti-tumor potential of type-I NKT cells against CD1d-positive and CD1dnegative tumors in humans. Clin Immunol. 2011;140(2):119-129.

12. Metelitsa LS, et al. Natural killer T cells infiltrate neuroblastomas expressing the chemokine CCL2. J Exp Med. 2004;199(9):1213-1221.

13. Tachibana T, et al. Increased intratumor V $\alpha 24$ positive natural killer $\mathrm{T}$ cells: a prognostic factor for primary colorectal carcinomas. Clin Cancer Res. 2005;11(20):7322-7327.

14. Molling JW, et al. Low levels of circulating invariant natural killer $\mathrm{T}$ cells predict poor clinical out- come in patients with head and neck squamous cell carcinoma. JClin Oncol. 2007;25(7):862-868.

15. Cariani E, et al. Immunological and molecular correlates of disease recurrence after liver resection for hepatocellular carcinoma. PLoS One. 2012;7(3):e32493.

16. Tahir SM, et al. Loss of IFN- $\gamma$ production by invariant NK T cells in advanced cancer. J Immunol. 2001;167(7):4046-4050.

17. Dhodapkar MV, et al. A reversible defect in natural killer $\mathrm{T}$ cell function characterizes the progression of premalignant to malignant multiple myeloma. JExp Med. 2003;197(12):1667-1676.

18. Heczey A, et al. Invariant NKT cells with chimeric antigen receptor provide a novel platform for safe and effective cancer immunotherapy. Blood. 2014;124(18):2824-2833.

19. Kalos M, June CH. Adoptive T cell transfer for cancer immunotherapy in the era of synthetic biology. Immunity. 2013;39(1):49-60.

20. Dotti G, Gottschalk S, Savoldo B, Brenner MK. Design and development of therapies using chimeric antigen receptor-expressing T cells. Immunol Rev. 2014;257(1):107-126.

21. Cohen NR, et al. Shared and distinct transcriptional programs underlie the hybrid nature of iNKT cells. Nat Immunol. 2013;14(1):90-99.

22. Constantinides MG, Bendelac A. Transcriptional regulation of the NKT cell lineage. Curr Opin Immunol. 2013;25(2):161-167.

23. Gapin L, Matsuda JL, Surh CD, Kronenberg M. NKT cells derive from double-positive thymocytes that are positively selected by CD1d. Nat Immunol. 2001;2(10):971-978.

24. Savage AK, et al. The transcription factor PLZF directs the effector program of the NKT cell lineage. Immunity. 2008;29(3):391-403.

25. Matsuda JL, et al. Homeostasis of V $\alpha 14 \mathrm{i}$ NKT cells. Nat Immunol. 2002;3(10):966-974.

26. Baev DV, et al. Distinct homeostatic requirements of $\mathrm{CD}^{+}{ }^{+}$and $\mathrm{CD} 4^{-}$subsets of $\mathrm{V} \alpha 24$ invariant natural killer T cells in humans. Blood. 2004;104(13):4150-4156.

27. Lee PT, Benlagha K, Teyton L, Bendelac A. Distinct functional lineages of human $\mathrm{V} \alpha 24$ natural killer T cells. J Exp Med. 2002;195(5):637-641.

28. Metelitsa LS, et al. Human NKT cells mediate antitumor cytotoxicity directly by recognizing target cell CD1d with bound ligand or indirectly by producing IL-2 to activate NK cells. J Immunol. 2001;167(6):3114-3122.

29. Loza MJ, Metelitsa LS, Perussia B. NKT and T cells: coordinate regulation of NK-like phenotype and cytokine production. Eur J Immunol. 2002;32(12):3453-3462.
30. Sallusto F, Geginat J, Lanzavecchia A. Central memory and effector memory $\mathrm{T}$ cell subsets: function, generation, and maintenance. Annu Rev Immunol. 2004;22:745-763.

31. D'Andrea A, et al. Neonatal invariant V $\alpha 24^{+} \mathrm{NKT}$ lymphocytes are activated memory cells. Eur J Immunol. 2000;30(6):1544-1550.

32. Kronenberg M, Gapin L. The unconventional lifestyle of NKT cells. Nat Rev Immunol. 2002;2(8):557-568.

33. Eger KA, Sundrud MS, Motsinger AA, Tseng M, Van KL, Unutmaz D. Human natural killer T cells are heterogeneous in their capacity to reprogram their effector functions. PLoS One. 2006;1:e50.

34. DelaRosa O, et al. V $\alpha 24^{+}$NKT cells are decreased in elderly humans. Exp Gerontol. 2002;37(2-3):213-217.

35. Okada R, Kondo T, Matsuki F, Takata H, Takiguchi M. Phenotypic classification of human $\mathrm{CD}^{+}$ $\mathrm{T}$ cell subsets and their differentiation. Int Immunol. 2008;20(9):1189-1199.

36. Graef P, et al. Serial transfer of single-cellderived immunocompetence reveals stemness of CD8(+) central memory T cells. Immunity. 2014;41(1):116-126.

37. Wang $X$, et al. Phenotypic and functional attributes of lentivirus-modified CD19specific human $\mathrm{CD}^{+}$central memory T cells manufactured at clinical scale. J Immunother. 2012;35(9):689-701.

38. Sommermeyer D, et al. Chimeric antigen receptor-modified $\mathrm{T}$ cells derived from defined CD8 CD4 subsets confer superior antitumor reactivity in vivo. Leukemia. 2016;30(2):492-500.

39. Carr T, Krishnamoorthy V, Yu S, Xue HH, Kee BL, Verykokakis M. The transcription factor lymphoid enhancer factor 1 controls invariant natural killer T cell expansion and Th2-type effector differentiation. J Exp Med. 2015;212(5):793-807.

40. van den Heuvel MJ, Garg N, Van KL, Haeryfar SM. NKT cell costimulation: experimental progress and therapeutic promise. Trends $\mathrm{Mol} \mathrm{Med}$. 2011;17(2):65-77.

41. Liu D, et al. Medulloblastoma expresses CD1d and can be targeted for immunotherapy with NKT cells. Clin Immunol. 2013;149(1):55-64.

42. Exley MA, et al. Selective activation, expansion, and monitoring of human iNKT cells with a monoclonal antibody specific for the TCR $\alpha$-chain CDR3 loop. Eur J Immunol. 2008;38(6):1756-1766.

43. Porter DL, Levine BL, Kalos M, Bagg A, June $\mathrm{CH}$. Chimeric antigen receptor-modified T cells in chronic lymphoid leukemia. $N$ Engl J Med. 2011;365(8):725-733. 
44. Grupp SA, et al. Chimeric antigen receptor-modified T cells for acute lymphoid leukemia. N EnglJ Med. 2013;368(16):1509-1518.

45. Brentjens RJ, et al. CD19-targeted T cells rapidly induce molecular remissions in adults with chemotherapy-refractory acute lymphoblastic leukemia. Sci Transl Med. 2013;5(177):177ra138.

46. Kochenderfer JN, et al. B-cell depletion and remissions of malignancy along with cytokineassociated toxicity in a clinical trial of anti-CD19 chimeric-antigen-receptor-transduced T cells. Blood. 2012;119(12):2709-2720.

47. Savoldo B, et al. CD28 costimulation improves expansion and persistence of chimeric antigen receptor-modified $\mathrm{T}$ cells in lymphoma patients. J Clin Invest. 2011;121(5):1822-1826.

48. Xu Y, et al. Closely related T-memory stem cells correlate with in vivo expansion of CAR.CD19-T cells and are preserved by IL-7 and IL-15. Blood. 2014;123(24):3750-3759.

49. Ara T, et al. Critical role of STAT3 in IL-6-mediated drug resistance in human neuroblastoma. Cancer Res. 2013;73(13):3852-3864.

50. Gattinoni L, et al. Acquisition of full effector function in vitro paradoxically impairs the in vivo antitumor efficacy of adoptively transferred CD8 ${ }^{+}$ T cells. JClin Invest. 2005;115(6):1616-1626.

51. Klebanoff CA, et al. Central memory self/ tumor-reactive $\mathrm{CD}^{+} \mathrm{T}$ cells confer superior antitumor immunity compared with effector memory T cells. Proc Natl Acad Sci U S A. 2005;102(27):9571-9576.

52. Der Vliet HJ, et al. Human natural killer T cells acquire a memory-activated phenotype before birth. Blood. 2000;95(7):2440-2442.

53. Constantinides MG, Picard D, Savage AK, Bendelac A. A naive-like population of human CD1drestricted $\mathrm{T}$ cells expressing intermediate levels of promyelocytic leukemia zinc finger. J Immunol. 2011;187(1):309-315.

54. Vivier E, Ugolini S, Blaise D, Chabannon C, Brossay L. Targeting natural killer cells and natural killer T cells in cancer. Nat Rev Immunol. 2012;12(4):239-252.

55. Uldrich AP, et al. NKT cell stimulation with glycolipid antigen in vivo: costimulation-dependent expansion, Bim-dependent contraction, and hyporesponsiveness to further antigenic challenge. JImmunol. 2005;175(5):3092-3101.

56. Acuto O, Michel F. CD28-mediated co-stimulation: a quantitative support for TCR signalling. Nat Rev Immunol. 2003;3(12):939-951.

57. Kroczek RA, Mages HW, Hutloff A. Emerging paradigms of T-cell co-stimulation. Curr Opin Immunol. 2004;16(3):321-327.

58. Redmond WL, Ruby CE, Weinberg AD. The role of OX40-mediated co-stimulation in T-cell activation and survival. Crit Rev Immunol. 2009;29(3):187-201.

59. Croft M. Control of immunity by the TNFR-related molecule OX40 (CD134). Annu Rev Immunol. 2010;28:57-78.

60. Lynch DH. The promise of 4-1BB (CD137)-mediated immunomodulation and the immunotherapy of cancer. Immunol Rev. 2008;222:277-286.

61. Suhoski MM, et al. Engineering artificial antigen-presenting cells to express a diverse array of co-stimulatory molecules. Mol Ther. 2007;15(5):981-988.

62. Thomas AK, Maus MV, Shalaby WS, June $\mathrm{CH}$, Riley JL. A cell-based artificial antigen-presenting cell coated with anti-CD3 and CD28 antibodies enables rapid expansion and long-term growth of CD4 T lymphocytes. Clin Immunol. 2002;105(3):259-272.

63. Maus MV, et al. Ex vivo expansion of polyclonal and antigen-specific cytotoxic $\mathrm{T}$ lymphocytes by artificial APCs expressing ligands for the T-cell receptor, CD28 and 4-1BB. Nat Biotechnol. 2002;20(2):143-148.

64. Barakonyi A, et al. Cutting edge: engagement of CD160 by its HLA-C physiological ligand triggers a unique cytokine profile secretion in the cytotoxic peripheral blood NK cell subset. J Immunol. 2004;173(9):5349-5354.

65. King MA, et al. Human peripheral blood leucocyte non-obese diabetic-severe combined immunodeficiency interleukin-2 receptor gamma chain gene mouse model of xenogeneic graft-versus-host-like disease and the role of host major histocompatibility complex. Clin Exp Immunol. 2009;157(1):104-118.

66. Chan WK, et al. Chimeric antigen receptorredirected CD45RA-negative T cells have potent antileukemia and pathogen memory response without graft-versus-host activity. Leukemia. 2015;29(2):387-395.

67. Ramos CA, Heslop HE, Brenner MK. CAR-T cell therapy for lymphoma. Annu Rev Med. 2016;67:165-183.

68. Pegram HJ, Smith EL, Rafiq S, Brentjens RJ. CAR therapy for hematological cancers: can success seen in the treatment of B-cell acute lymphoblastic leukemia be applied to other hematological malignancies? Immunotherapy. 2015;7(5):545-561.

69. Pillai AB, George TI, Dutt S, Teo P, Strober S. Host NKT cells can prevent graft-versus-host disease and permit graft antitumor activity after bone marrow transplantation. J Immunol. 2007;178(10):6242-6251.

70. Morris ES, et al. NKT cell-dependent leukemia eradication following stem cell mobilization with potent G-CSF analogs. JClin Invest. 2005;115(11):3093-3103.

71. de Lalla C, et al. Invariant NKT cell reconstitution in pediatric leukemia patients given HLAhaploidentical stem cell transplantation defines distinct $\mathrm{CD}^{+}$and $\mathrm{CD}^{-}{ }^{-}$subset dynamics and correlates with remission state. JImmunol. 2011;186(7):4490-4499.

72. Casorati G, de Lalla C, Dellabona P. Invariant natural killer $\mathrm{T}$ cells reconstitution and the control of leukemia relapse in pediatric haploidentical hematopoietic stem cell transplantation. Oncoimmunology. 2012;1(3):355-357.

73. Pulè MA, Straathof KC, Dotti G, Heslop HE, Rooney CM, Brenner MK. A chimeric T cell antigen receptor that augments cytokine release and supports clonal expansion of primary human $\mathrm{T}$ cells. Mol Ther. 2005;12(5):933-941.

74 . Vera J, et al. T lymphocytes redirected against the kappa light chain of human immunoglobulin efficiently kill mature B lymphocyte-derived malignant cells. Blood. 2006;108(12):3890-3897.

75. Jena B, et al. Chimeric antigen receptor (CAR)specific monoclonal antibody to detect CD19specific T cells in clinical trials. PLoS One. 2013;8(3):e57838.

76. Torikai H, et al. Toward eliminating HLA class I expression to generate universal cells from allogeneic donors. Blood. 2013;122(8):1341-1349. 